\title{
A COMPREHENSIVE METHOD OF ESTIMATING ELECTRIC FIELDS FROM VECTOR MAGNETIC FIELD AND DOPPLER MEASUREMENTS
}

\author{
Maria D. Kazachenko, George H. Fisher, and Brian T. Welsch \\ Space Sciences Laboratory, UC Berkeley, CA 94720, USA; kazachenko@ssl.berkeley.edu \\ Received 2014 April 15; accepted 2014 September 4; published 2014 October 9
}

\begin{abstract}
Photospheric electric fields, estimated from sequences of vector magnetic field and Doppler measurements, can be used to estimate the flux of magnetic energy (the Poynting flux) into the corona and as time-dependent boundary conditions for dynamic models of the coronal magnetic field. We have modified and extended an existing method to estimate photospheric electric fields that combines a poloidal-toroidal decomposition (PTD) of the evolving magnetic field vector with Doppler and horizontal plasma velocities. Our current, more comprehensive method, which we dub the "PTD-Doppler-FLCT Ideal" (PDFI) technique, can now incorporate Doppler velocities from non-normal viewing angles. It uses the FISHPACK software package to solve several two-dimensional Poisson equations, a faster and more robust approach than our previous implementations. Here, we describe systematic, quantitative tests of the accuracy and robustness of the PDFI technique using synthetic data from anelastic MHD (ANMHD) simulations, which have been used in similar tests in the past. We find that the PDFI method has less than $1 \%$ error in the total Poynting flux and a $10 \%$ error in the helicity flux rate at a normal viewing angle $(\theta=0)$ and less than $25 \%$ and $10 \%$ errors, respectively, at large viewing angles $\left(\theta<60^{\circ}\right)$. We compare our results with other inversion methods at zero viewing angle and find that our method's estimates of the fluxes of magnetic energy and helicity are comparable to or more accurate than other methods. We also discuss the limitations of the PDFI method and its uncertainties.
\end{abstract}

Key words: magnetic fields - Sun: evolution - Sun: flares - Sun: fundamental parameters Sun: photosphere - sunspots

Online-only material: color figures

\section{INTRODUCTION}

Energy from magnetic fields on the Sun powers nearly all manifestations of solar activity, from heating in active regions and the solar wind to dramatic events like flares and coronal mass ejections (CMEs). Quantitative studies of the flow of magnetic energy through the solar atmosphere require a knowledge of both the magnetic and electric field vectors. The goal of this paper is to show how time sequences of vector magnetic field maps (vector magnetograms), along with other observational constraints such as Doppler flow measurements, can be used in a practical way to construct maps of the electric field vector. Knowing both the electric and magnetic field, we can find the Poynting flux of electromagnetic energy and the flux of relative magnetic helicity, important quantities that describe how magnetic energy is transported, stored, and released in the solar atmosphere.

Past attempts to determine the electric field distribution on the Sun have followed two approaches: (1) direct spectroscopic measurements of the electric field using the linear Stark effect, (2) indirect determinations of the electric field by using Faraday's law, relating the temporal derivative of the magnetic field to the curl of the electric field. Wien (1916) was the first to suggest measuring the electric field of solar plasma directly using the Stark effect. In the 1980s, some attempts were made to measure the electric field at the Sun using helium and silicon spectra, finding an electric field of $700 \mathrm{~V} \mathrm{~cm}^{-1}$ (Davis 1977) and $300 \mathrm{~V} \mathrm{~cm}^{-1}$ (Jordan et al. 1980). Later, examining Starkbroadened Paschen emission lines in hydrogen, Moran \& Foukal (1991) found an upper limit of $5-10 \mathrm{~V} \mathrm{~cm}^{-1}$. In the same work, they pointed out that the direct measurement of the electric field using the Stark effect is difficult because of the low sensitivity of the measurements.
Indirect determinations of the electric field have been considerably more successful. The implementation of local-correlation tracking techniques (LCT; e.g., November \& Simon 1988; Berger et al. 1995; Hurlburt et al. 1995; Fisher \& Welsch 2008), applied to time sequences of magnetograms allows one to determine a "pattern motion" velocity of the line-of-sight component of the magnetic field in the plane of the magnetogram (Démoulin $\&$ Berger 2003). Interpreting the pattern motion as a plasma velocity and assuming an ideal MHD Ohm's law for the electric field $(c \mathbf{E}=-\mathbf{V} \times \mathbf{B})$ allows one to determine some of the information about the velocity field, and hence by assumption, the electric field. These techniques can be improved by explicitly incorporating the normal component of the magnetic induction equation into the solution for the components of the velocity $\mathbf{V}$ (Kusano et al. 2002; Welsch et al. 2004; Longcope 2004; Schuck 2006, 2008; Chae \& Sakurai 2008), resulting in improvements in the velocity inversion (Welsch et al. 2007).

More recently, we have developed inversion techniques for the electric field itself rather than the velocity field based on a poloidal-toroidal decomposition (PTD) of the magnetic field and its time derivative. These techniques use Faraday's law and other theoretical and observational constraints to determine the electric field (Fisher et al. 2010, 2012). Inversions for the electric field instead of the velocity field offer a few distinct advantages. First, the PTD method incorporates additional information from evolution of the horizontal magnetic field (the field parallel to the photosphere). This is used to make an estimate of the normal electric field, independent of the horizontal electric field inferred by methods that derive $\mathbf{V}$ from the normal component of the induction equation. Second, in regions where the magnetic field is relatively weak or uncertain, the determination of the velocity field is especially ill-posed. For instance, outside of active regions, fluctuations in the normal magnetic field can 
be dominated by noise, resulting in a wildly varying and unphysical behavior in the inferred $\mathbf{V}$. Complex masking and filtering techniques must be applied post facto to suppress this behavior. The solutions for the electric field, on the other hand, may contain small errors in regions with small magnetic field strength, but the solutions vary smoothly within regions containing both strong and weak magnetic fields. Third, if a model for non-ideal effects is included in the electric field $\mathbf{E}$ assumed to drive the change in $\mathbf{B}$, they can be captured by the PTD solutions while the velocity formalism may give spurious results.

Fisher et al. (2010) described in detail the PTD techniques necessary to find the "inductive" electric field that satisfies Faraday's law given the spatial distribution of the temporal derivative of the magnetic field measured across a region of the solar photosphere. They also noted that the resulting solutions are not unique in that gradients of arbitrary scalar functions could be added to the PTD solutions without affecting Faraday's law. They presented several approaches to specify the electric field uniquely, including requiring the electric field to be perpendicular to the magnetic field, consistent with the ideal Ohm's law. Fisher et al. (2012) demonstrated that by adding to the PTD solutions the gradients of scalar functions that are constructed to match the electric field near polarity inversion lines (PILs), the accuracy of electric field reconstructions could be substantially improved beyond all of the velocity inversion techniques considered in the comparative study of Welsch et al. (2007). To match the electric field near PILs, one can use measurements of the Doppler velocity and the transverse magnetic field distribution in those regions.

In this paper, we build on Fisher et al. (2012) results in a number of ways with the goal of carefully describing and validating a practical implementation of the introduced methods that could be used to routinely find the electric field from sequences of vector magnetogram observations, such as those available from the Helioseismic and Magnetic Imager (HMI) (Schou et al. 2012; Scherrer et al. 2012) on NASA's SDO mission. First, we adopt the use of the FISHPACK software library for Helmholtz equations (Schwarztrauber 1975), developed at NCAR in the 1970s, for solving the two-dimensional Poisson equations that are at the heart of the PTD inversion technique. This software, based on the cyclic reduction technique, is very efficient and explicitly allows for the specification of normalderivative (Neumann) boundary conditions that are consistent with our desired boundary conditions on the electric field at the edges of vector magnetogram images. Second, we develop the ability to incorporate line-of-sight Doppler measurements that are taken at non-normal viewing angles, appropriate for vector magnetograms that are not at disk center, to determine electric fields near PILs. Finally, to validate our inversion methods, we analyze the performance of our techniques comparing electric field inversions with a test case from an MHD simulation where the true electric field is known (Welsch et al. 2007). Besides the electric field, we compare derived values of the Poynting flux and the relative helicity fluxes with the known values from the test case. Finally, in the Appendix, we describe how we adapt our electric field solution techniques to spherical polar coordinates appropriate for larger fields of view on the Sun.

The remainder of the paper is structured as follows. In Section 2, we describe the electric field inversion technique, starting from the basic PTD formalism and also improvements that account for a non-zero viewing angle. In Section 3, we describe how to calculate Poynting and helicity fluxes using

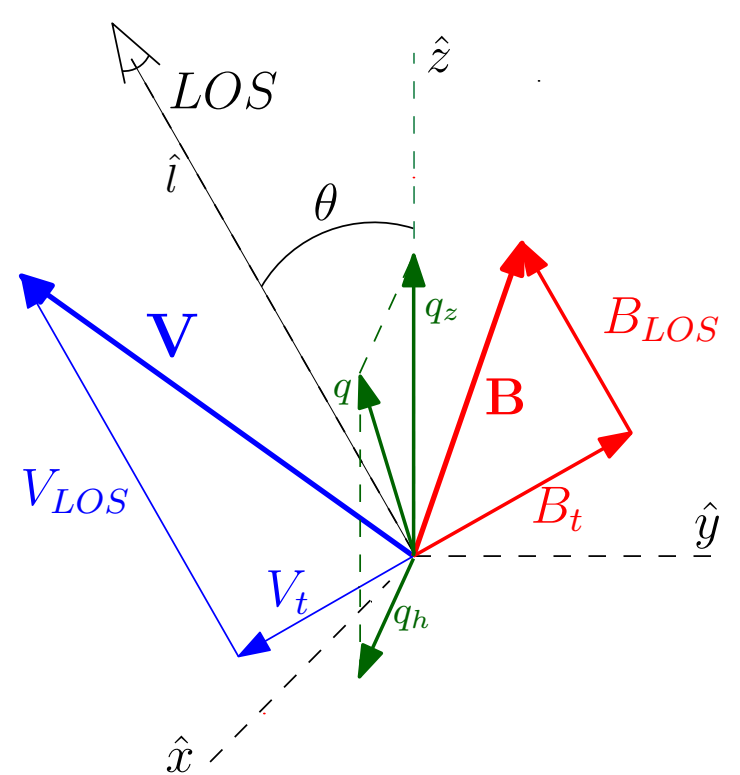

Figure 1. Illustration of the relationship between the LOS direction and the transverse and LOS components of $\mathbf{B}$ and $\mathbf{V}$ and $\hat{\mathbf{q}}$.

(A color version of this figure is available in the online journal.)

our derived electric field. This includes a decomposition of the Poynting flux into fluxes of potential and free magnetic energies. In Section 4, we perform tests of the electric field inversion method using the ANMHD simulation test case and provide validation metrics that quantify how well the inversion recovers the true solution. Finally, in Section 5, we summarize the strengths and weaknesses of our technique and describe how it will be used to analyze HMI vector magnetograms.

\section{FINDING ELECTRIC FIELDS}

\subsection{The Inductive PTD Electric Field: $\boldsymbol{E}^{P}$}

PTD allows one to estimate the photospheric electric field vector from the evolution of the photospheric magnetic field vector. Here we present a brief synopsis of the PTD method (Fisher et al. 2010), plus improvements we have made since that article was published.

The fundamental idea of PTD is that the magnetic field vector $\mathbf{B}=\left(B_{x}, B_{y}, B_{z}\right)$ has a solenoidal nature and hence can be specified by two scalar functions, the "poloidal" and "toroidal" potentials (Chandrasekhar 1961; Moffatt 1978), which we denote as $\mathcal{B}$ and $\mathcal{J}$ following the notation used in descriptions of anelastic MHD that employ these potentials (Lantz \& Fan 1999):

$$
\mathbf{B}=\nabla \times \nabla \times \mathcal{B} \hat{\mathbf{z}}+\nabla \times \mathcal{J} \hat{\mathbf{z}} .
$$

Here we consider a locally Cartesian coordinate system $(x, y, z)$, that has its $z$ axis oriented perpendicular to the photosphere, with the positive direction away from the Sun's center (see Figure 1; the spherical case is described in the Appendix). We use subscript $z$ to denote vector components or derivatives in the vertical direction, and subscript $h$ to denote vector components or derivatives in the horizontal directions, i.e., parallel to the photosphere.

Taking the partial time derivative of Equation (1), one finds

$$
\dot{\mathbf{B}}=\nabla \times \nabla \times \dot{\mathcal{B}} \hat{\mathbf{z}}+\nabla \times \dot{\mathcal{J}} \hat{\mathbf{z}}
$$

By examining the $z$ component of Equation (2), its horizontal divergence, and the $z$ component of its curl, we find three 
two-dimensional Poisson equations for the unknown functions $\dot{\mathcal{B}}, \partial \dot{\mathcal{B}} / \partial z$, and $\dot{\mathcal{J}}$ in terms of known physical quantities:

$$
\begin{gathered}
-\dot{B}_{z}=\nabla_{h}^{2} \dot{\mathcal{B}} \\
\nabla_{h} \cdot \dot{\mathbf{B}}_{h}=\frac{\partial \dot{B}_{x}}{\partial x}+\frac{\partial \dot{B}_{y}}{\partial y}=\nabla_{h}^{2}\left(\frac{\partial \dot{\mathcal{B}}}{\partial z}\right) \\
-\hat{\mathbf{z}} \cdot\left(\nabla \times \dot{\mathbf{B}}_{h}\right)=-\frac{4 \pi}{c} \dot{J}_{z}=\frac{\partial \dot{B}_{x}}{\partial y}-\frac{\partial \dot{B}_{y}}{\partial x}=\nabla_{h}^{2} \dot{\mathcal{J}}
\end{gathered}
$$

where $\nabla_{h}^{2}=\left(\partial^{2}\right) /\left(\partial x^{2}\right)+\left(\partial^{2}\right) /\left(\partial y^{2}\right)$, and the Ampère's law for vertical current $\dot{J}_{z}$ has been used in Equation (5). Comparing Faraday's law with Equation (2), we find

$$
-c \nabla \times \mathbf{E}=\dot{\mathbf{B}}=\nabla \times \nabla \times \dot{\mathcal{B}} \hat{\mathbf{z}}+\nabla \times \dot{\mathcal{J}} \hat{\mathbf{z}}
$$

For future use, we can rewrite this result as

$$
-c \nabla \times \mathbf{E}=\dot{\mathbf{B}}=-\nabla_{h}^{2} \dot{\mathcal{B}} \hat{\mathbf{z}}+\nabla_{h} \frac{\partial \dot{\mathcal{B}}}{\partial z}+\nabla \times \dot{\mathcal{J}} \hat{\mathbf{z}}
$$

Uncurling Equation (6), we derive the electric field $\mathbf{E}$ in terms of $\dot{\mathcal{B}}$ and $\dot{\mathcal{J}}$ :

$$
\begin{aligned}
c \mathbf{E} & =c\left(E_{x}, E_{y}, E_{z}\right)=-\nabla \times \dot{\mathcal{B}} \hat{\mathbf{z}}-\dot{\mathcal{J}} \hat{\mathbf{z}}-\nabla \psi \\
& \equiv \underbrace{c \mathbf{E}^{\mathbf{P}}}_{\text {inductive }}-\underbrace{\nabla \psi}_{\text {non-inductive }} .
\end{aligned}
$$

Here, $\mathbf{E}^{\mathbf{P}}$ is the purely inductive contribution to the electric field determined by PTD potentials, $\dot{\mathcal{B}}$ and $\dot{\mathcal{J}}$. Within this article, $\mathbf{E}^{\mathbf{P}}$ will be referred to as the P solution, or the PTD electric field, where superscript "P" stands for the PTD method. At this point, two comments can provide some insight into the nature of $\mathbf{E}^{\mathbf{P}}$ : (1) the horizontal components of $\mathbf{E}^{\mathbf{P}}$ only depend upon $\dot{\mathcal{B}}$, which was derived from $B_{z}$; and (2) the vertical component of $\mathbf{E}^{\mathbf{P}}$ only depends upon $\mathcal{J}$, which is derived from $\dot{J}_{z}$. The component of the total electric field arising from $-\nabla \psi$ is the non-inductive contribution, for which the PTD solution to Faraday's law, Equation (7), reveals no information. For the simulation data analyzed by Welsch et al. (2007), Fisher et al. (2010) demonstrated that when $(-\nabla \psi=0)$, i.e., when non-inductive contributions to the electric field are ignored, $\mathbf{E}^{P}$ does a poor job of representing the actual electric field. The key to a more accurate reconstruction of the electric field is incorporating other physical constraints, and/or additional observational information into the solutions for $\nabla \psi$. Procedures for doing this will be described further below. However, we first complete our discussion for obtaining the full $\mathbf{E}^{P}$ (PTD) solution.

To find $\dot{\mathcal{B}}$ and $\dot{\mathcal{J}}$, and hence the $\mathbf{E}^{P}$ solution, we must solve the three Poisson equations, (3)-(5), with well-posed boundary conditions. To permit a net gradient in each potential over the field of view, we choose Neumann boundary conditions

$$
\begin{gathered}
\frac{\partial \dot{\mathcal{B}}}{\partial n}=0, \\
\frac{\partial}{\partial n}\left(\frac{\partial \dot{\mathcal{B}}}{\partial z}\right)=\dot{B}_{n}-\frac{\partial \dot{\mathcal{J}}}{\partial s}, \quad \text { and } \\
\frac{\partial \dot{\mathcal{J}}}{\partial n}=-\dot{B}_{s}+\frac{\partial}{\partial s}\left(\frac{\partial \dot{\mathcal{B}}}{\partial z}\right),
\end{gathered}
$$

where subscript $n$ denotes components or derivatives in the direction of the outward normal to magnetogram's boundary and subscript $s$ denotes components or derivatives in the counterclockwise direction along the magnetogram boundary. The first boundary condition, Equation (9), implies that the tangential component of the electric field around the magnetogram boundary vanishes, implying that the average value of $\dot{B}_{z}$ within the magnetogram is zero. If the average value of $\dot{B}_{z}$ is not zero (i.e., a change in the flux balance, or monopole term), then we can add a correction term to the electric field post facto (see Appendix $\mathrm{C}$ of Fisher et al. 2010). The second and third boundary conditions, Equations (10) and (11), derived from evaluating Equation (7) at the magnetogram boundary, are degenerate, i.e., there is a family of coupled non-zero solutions, $\dot{\mathcal{J}}$ and $(\partial \dot{\mathcal{B}} / \partial z)$, which correspond to zero time derivative of the horizontal magnetic field:

$$
\frac{\partial}{\partial x}\left(\frac{\partial \dot{\mathcal{B}}}{\partial z}\right)=-\frac{\partial \dot{\mathcal{J}}}{\partial y}
$$

and

$$
\frac{\partial}{\partial y}\left(\frac{\partial \dot{\mathcal{B}}}{\partial z}\right)=\frac{\partial \dot{\mathcal{J}}}{\partial x} .
$$

Since the solutions to these Cauchy-Riemann equations each satisfy the two-dimensional Laplace equation, they can be added to solutions of Equations (4) and (5) without changing the time derivative of the horizontal field on the boundary. This means that there is some freedom to specify the solutions of $\dot{\mathcal{J}}$ or $(\partial \dot{\mathcal{B}} / \partial z)$ at the boundary. In practice, this means that one could choose to set the derivative parallel to the boundary of one of these two functions to zero while still obeying the coupled boundary conditions (10) and (11). To remove the coupling between the boundary conditions (10) and (11), we choose $\partial \dot{\mathcal{J}} / \partial s=0$ along the magnetogram boundary, meaning that $\dot{\mathcal{J}}$ is assumed uniform along the magnetogram boundary. (This implies no change in the net signed vertical current through the field of view.) This also allows us to set the value of $E_{z}$ for $\mathbf{E}^{P}$ to zero at the magnetogram boundary by simply subtracting the boundary value from the entire solution for $\dot{\mathcal{J}}$. Choosing the condition $\partial \dot{\mathcal{J}} / \partial s=0$ also means that the solution of the Poisson equations must be done in a certain order. First, the solution for $(\partial \dot{\mathcal{B}} / \partial z)$ must be obtained, then the solution for $\dot{\mathcal{J}}$ can be obtained using the variation of $(\partial \dot{\mathcal{B}} / \partial z)$ along the magnetogram boundary to specify the normal derivative of $\dot{\mathcal{J}}$. The solution for $\dot{\mathcal{B}}$ is independent of the other two solutions and so can be obtained either before or after obtaining the other two solutions.

When solving Poisson equations (3)-(5) and boundary conditions (9)-(11) numerically, it is important to implement a fast and robust numerical scheme, especially when dealing with large magnetogram data sets. In the past, Fisher et al. (2012, 2010) used the Newton-Krylov technique adapted from the RADMHD code (Abbett 2007). Its main disadvantage is slow computational speed and poor robustness when applied to this particular problem. In this article, we adopt FISHPACK, a fast and robust collection of Fortran subprograms developed at NCAR (Schwarztrauber 1975). FISHPACK applies the cyclic reduction technique (Sweet 1974) and the standard five-point finite difference approximation for the Laplacian to solve the twodimensional Helmholtz equation in Cartesian or spherical (see the Appendix) coordinates. This software was designed to solve Helmholtz equations (the Poisson equation is a special case of the Helmholtz equation) with Neumann, Dirichlet, or periodic boundary conditions and can use either a centered or staggered 
grid. To convert our equations into a form that is compatible with the FISHPACK subroutine HWSCRT, we use centered, secondorder accurate finite differences to approximate first derivatives in $x$ and $y$ and the centered five-point expression for the Laplacian of a function $f$ in a Cartesian, two-dimensional geometry:

$$
\begin{gathered}
\left.\frac{\partial f}{\partial x}\right|_{i, j}=\left(f_{i+1, j}-f_{i-1, j}\right) /(2 \Delta x), \\
\left.\frac{\partial f}{\partial y}\right|_{i, j}=\left(f_{i, j+1}-f_{i, j-1}\right) /(2 \Delta y), \\
\nabla_{h}^{2} f=\left(f_{i+1, j}+f_{i-1, j}+f_{i, j+1}+f_{i, j-1}-4 f_{i, j}\right) /(\Delta x)^{2},
\end{gathered}
$$

where in the last equation, we assume $\Delta x=\Delta y$. Similar to spatial differences, to evaluate the time derivative of function $f_{i, j}$ at time $t_{k}, \dot{f}_{i, j}\left(t_{k}\right)$, the source terms (the left-hand sides) of Equations (3)-(5), we use the centered finite difference approximation

$$
\dot{f}_{i, j}\left(t_{k}\right)=\left(f_{i, j}\left(t_{k+1}\right)-f_{i, j}\left(t_{k-1}\right)\right) /(2 \Delta t),
$$

where $\Delta t$ is the time step between two consecutive frames $\Delta t=t_{k+1}-t_{k}$. Once a solution for $\dot{\mathcal{B}}$ has been obtained, the electric field components $E_{x}^{P}$ and $E_{y}^{P}$ are computed by using the finite derivative approximations (Equations (14) and (15)) in the expression for $-\nabla \times \dot{\mathcal{B}} \hat{\mathbf{z}}$; for $E_{z}^{P}$ we use the solution $-\dot{\mathcal{J}}$. We add a single layer of ghost zones around the periphery of each solution domain to ensure that the Neumann boundary conditions are obeyed when the above finite difference expressions are used. Given the known Neumann (normal first derivative) boundary conditions (Equations (9)-(11)) and the interior solution points that are returned from HWSCRT, using the expressions in Equations (14) and (15), we then determine the values of the solution variables in the ghost zone layers. This procedure leaves the four corners of the ghost zone values undefined; for cosmetic reasons, when displaying the solution, we assume each corner value is the average of its two nearest-neighbor ghost zone values.

As Schwarztrauber (1975) points out, it is possible to specify Neumann boundary conditions that are incompatible with some Poisson equations. Their approach to this quandary is to add a constant value to the source term of the Poisson equation, with that value varied until the best possible fit to the boundary values can be obtained. This offset value is returned by HWSCRT subroutine as the PERTRB variable, which we record along with the solution itself. The value of PERTRB is then monitored to ensure that it remains small compared to typical values of the source term of our Poisson equations.

Finite difference approximations for the first derivative (Equations (14) and (15)) and the Laplacian (Equation (16)) introduce incompatibilities, since the five-point Laplacian implicitly assumes that first derivatives are evaluated at half-integer grid locations. These incompatibilities could be eliminated by using a staggered grid in which electric field variables are defined at cell edges, while magnetic field values are defined at cell centers. However, we have found that the resulting errors are usually small, and the introduction of a staggered grid introduces a number of complications when adding the electric fields derived from the gradients of scalar potentials to the $\mathbf{E}^{P}$ solutions. Here, we have decided to accept these incompatibilities as a cost of using a centered grid where the electric field and magnetic field variables are assumed co-spatial. We may adopt a staggered grid in a future version of the software.
One case where the incompatibility errors may not be small, is if the solutions are dominated by high-frequency noise near the magnetogram boundaries because of, e.g., poor signal-to-noise ratios in areas of weak magnetic fields. In this case, we have found it useful to add "zero-padding" to the solution domain for a modest number of zones around the periphery of the observed magnetograms in which all magnetic field values and their time derivatives are set to zero, forcing the source terms of all of the Poisson equations to be zero within a thin ribbon inward of the computational boundary. The smoothness of the resulting Poisson equation solutions ensures that the incompatibilities of the finite difference approximations described above are not important in the boundary regions and therefore that the finite difference expressions for the Neumann boundary conditions are good approximations for the normal first derivatives.

\subsection{Enforcing the Condition $\boldsymbol{E} \cdot B=0$ with the Potential $\psi^{I}$}

One of the properties of the derived inductive PTD electric field $\mathbf{E}^{P}$ (Equation (8)) is that for most of the cases and even for ideal MHD case, $c \mathbf{E}=-\mathbf{V} \times \mathbf{B}$, that has $\mathbf{E}$ perpendicular to $\mathbf{B}$, the derived $\mathbf{E}^{P}$ is not perpendicular to $\mathbf{B}$. To resolve this inconsistency, in this section, we review the "iterative" method described in Section 3.2 of Fisher et al. (2010) and the changes that we have made to it since then. This technique will be used extensively in the discussions of other contributions to the electric field below.

The goal of the iterative method is to construct the gradient of an electric potential $\psi^{I}$ that obeys the constraint

$$
\nabla \psi^{I} \cdot \mathbf{B}=c \mathbf{E}^{P} \cdot \mathbf{B}
$$

for all points within the vector magnetogram domain. Once found, $\nabla \psi^{I}$ can then be subtracted from $\mathbf{E}^{P}$ to yield an electric field that is normal to $\mathbf{B}$, but that has the same curl as $\mathbf{E}^{P}$. In practice, and as described in Section 3.2 of Fisher et al. (2010), the procedure is to find two two-dimensional functions, $\psi^{I}(x, y)$ and $\partial \psi^{I} / \partial z(x, y)$ over the two-dimensional domain of the vector magnetogram such that

$$
\nabla_{h} \psi^{I} \cdot \hat{\mathbf{b}}_{h}+b_{z} \partial \psi^{I} / \partial z=c \mathbf{E}_{h}^{P} \cdot \hat{\mathbf{b}}_{h}+b_{z} c E_{z}^{P},
$$

where $\hat{\mathbf{b}}_{h}$ and $b_{z}$ are, respectively, the horizontal and vertical components of the unit vector $\hat{\mathbf{b}}$ that points in the direction of B. Once $\psi^{I}$ and $\partial \psi^{I} / \partial z$ have been found, then we define the quantity

$$
c \mathbf{E}^{P I}=c \mathbf{E}_{h}^{P}-\nabla_{h} \psi^{I}+\hat{\mathbf{z}}\left(c E_{z}^{P}-\partial \psi^{I} / \partial z\right),
$$

where $\mathbf{E}^{P I}$ then obeys both Faraday's law and the ideal constraint $\mathbf{E}^{P I} \cdot \mathbf{B}=0$.

We continue to follow the iterative method procedures described in Section 3.2 of Fisher et al. (2010) to determine $\psi^{I}$ and $\partial \psi^{I} / \partial z$, but with the following changes. First, in Step 4 of that procedure, in which a horizontal Poisson equation is solved to update the iterative guess for $\psi^{I}$, we now use the FISHPACK routine HWSCRT to solve the Poisson equation instead of using fast Fourier transform (FFT) techniques that implicitly assume periodic boundary conditions. We assume homogenous Neumann boundary conditions for $\psi^{I}$ such that $\partial \psi^{I} / \partial n=0$ on the outer boundary, meaning that the normal component of the horizontal electric field due to the scalar potential is zero on the outer boundary of the vector magnetogram. We use Equations (14) and (15) to approximate the components of the horizontal gradient of $\psi^{I}$ and Equation (16) to approximate the Laplacian. 
Ghost zone values are defined in a similar fashion as in the previous section for determining $\dot{\mathcal{B}}$. The solution for $\partial \psi^{I} / \partial z$, in Step 5 of the iterative procedure, is usually negligibly small at the outer boundary of the magnetogram provided that the horizontal components $\nabla_{h} \psi^{I}$ are also small there. This results in the desired property that $E_{z}^{P I}$ is essentially zero at the outer magnetogram boundary. An additional change we have made from Fisher et al. (2010) is that instead of evaluating an error criterion to end the iteration sequence, we have found it more efficient to simply specify a maximum number of iterations, typically chosen to be 25 (see discussion in Section 4.1).

An important point about the iterative method is that it can be applied not only to the PTD solution $\mathbf{E}^{P}$ itself, but to any solution for the electric field $\mathbf{E}$ that contains components parallel to $\mathbf{B}$ that one might like to minimize. In particular, we can apply the iterative method to solutions, which include both the PTD solution and additional electric field contributions from scalar potentials determined from Doppler measurements or correlation tracking results, as are described in further detail below. Once these interim solutions are obtained, the iterative method can then be applied as a final step to generate the scalar potential $\psi^{I}$ (and $\partial \psi^{I} / \partial z$ ) needed to satisfy $\mathbf{E} \cdot \mathbf{B}=0$.

Finally, we comment on the uniqueness of the solutions for $\psi^{I}$ found from the iterative method given a fixed input electric field E. As noted in Fisher et al. (2010), mathematically the constraint (18) does not result in a unique solution for $\psi^{I}$ and $\partial \psi^{I} / \partial z$. Empirically, however, we find that the iterative procedure of Section 3.2 of Fisher et al. (2010; and also with the modifications described here) consistently results in the same solution for $\psi^{I}$ and $\partial \psi^{I} / \partial z$ even when initialized with substantially different trial functions. We surmise that applying the iterative procedure itself imposes some additional constraints beyond (18), which we do not fully understand, but which then result in a unique solution $\psi^{I}$ for the input $\mathbf{E}$. In fact, given any $\mathbf{E}$ that is consistent with both Faraday's and Ohm's laws, an additional potential functions can be constructed that can be superposed without violating either constraint. So, while our solution method returns a unique answer, Faraday's and Ohm's laws do not, by themselves, fully constrain $\mathbf{E}$.

\subsection{Non-inductive Doppler and Fourier Local Correlation Tracking (FLCT) Electric Fields: $\boldsymbol{E}^{D}$ and $\boldsymbol{E}^{\boldsymbol{F} L C T}$}

When examining the first and second rows of Figure 2 of Fisher et al. (2012), comparing the actual components of the test simulation electric field with the same components of $\mathbf{E}^{P}$, as well as the actual and inverted vertical Poynting flux, one of the most obvious discrepancies is the far lower range of the Poynting flux values originating from the electric field inversion as compared to the true values (see Figure 3 in Fisher et al. 2012). The $x$ and $y$ components of $\mathbf{E}$ also show significant discrepancies, with the derived horizontal components of $\mathbf{E}$ being smaller in magnitude than the actual values. Is there some additional information about the horizontal electric field that can be determined from other observational data besides the magnetic field?

Estimating the transport of magnetic energy across the photosphere requires accurately reconstructing photospheric electric fields during flux emergence. From studying ANMHD simulations of an emerging bipolar magnetic configuration, we have found that by itself, Faraday's law applied to $\dot{B}_{z}$ does not completely capture significant aspects of horizontal electric fields present during magnetic flux emergence. Here we review two possible sources of electric fields that may not be reflected in solutions of Faraday's law: electric fields determined from Doppler measurements and those determined using correlation tracking methods. In the discussion below, we will first consider the computation of these electric fields from the conceptually easier vantage point of zero viewing angle, where the line of sight is normal to the solar surface (Sections 2.3.1 and 2.3.2). Once we have introduced all of the physical concepts, we will describe the modifications necessary to account for non-normal viewing angles (Section 2.3.3).

\subsubsection{Doppler Electric Fields: Normal Viewing Angle}

Certainly in flux-emergence regions where the vertical magnetic field is near zero (i.e., near PILs), a knowledge of the vertical velocity $V_{z}$ would yield an unambiguous determination of the horizontal electric field:

$$
c \mathbf{E}^{\mathrm{PIL}}=-V_{z} \hat{\mathbf{z}} \times \mathbf{B}_{h},
$$

if we can assume that an ideal MHD Ohm's law applies in the photosphere. Such measurements could be determined by Doppler velocity measurements viewed from directly above the active region. Figure 1 of Fisher et al. (2012) illustrates with a thought experiment that such a flux-emergence electric field might even have zero curl near the PIL, and yet, if the Doppler velocity component $V_{z}$ had a large amplitude along the PIL region, would still result in a very large electric field. One possible approach to incorporating such data would be to simply replace the PTD solution $\mathbf{E}^{P}$ with $\mathbf{E}^{\mathrm{PIL}}$ for those parts of the magnetogram near the PIL location. If one did this, however, the resulting solution near the PIL would not necessarily obey Faraday's law and furthermore could vary discontinuously and/ or unphysically as one crossed the boundary between regions where the two solution methods for $\mathbf{E}$ were applied.

The solution to this dilemma is to combine the two solutions in such a way that Faraday's law is obeyed everywhere in the magnetogram region, but that the non-inductive (curl-free) component of $\mathbf{E}^{\mathrm{PIL}}$ is added to $\mathbf{E}^{P}$ to provide a more realistic electric field estimate in flux emergence regions. To do this, we need two additional ingredients: (1) an estimate for $\mathbf{E}^{\mathrm{PIL}}$ that one can apply when one leaves the vicinity of the PIL, and (2) a technique for removing the inductive contribution from $\mathbf{E}^{\mathrm{PIL}}$ since $\mathbf{E}^{P}$ will contain all the needed information about Faraday's law.

Addressing the first of these ingredients, as one moves away from the PIL, Equation (21) becomes increasingly uncertain for two reasons: first, as the vertical component of the field becomes nonzero, it becomes increasingly possible for any existing flows parallel to the magnetic field to contribute to the Doppler signal without contributing to an electric field. Second, once $B_{z}$ is no longer zero, there is a contribution to the horizontal electric field from horizontal flows crossed into $B_{z} \hat{\mathbf{z}}$, which is not reflected in Equation (21). We adopt the ad-hoc solution of Fisher et al. (2012), namely that $\mathbf{E}^{\mathrm{PIL}}$ is multiplied by an attenuation or confidence factor, $w$, that reduces the amplitude of $\mathbf{E}^{\mathrm{PIL}}$ as the ratio $\left|B_{z}\right| /\left|B_{h}\right|$ increases above zero. We adopt the same functional form of $w$ introduced in Fisher et al. (2012), namely

$$
w=\exp \left[-\frac{1}{\sigma_{\mathrm{PIL}}^{2}}\left|\frac{B_{z}}{B_{h}}\right|^{2}\right],
$$

where $\sigma_{\text {PIL }}$ is an adjustable parameter that can be tuned by comparing with test case data where the electric field is known. The determination of the optimal value of $\sigma_{\mathrm{PIL}}$ for the ANMHD test case is discussed in Section 4.1. 
Addressing the second ingredient, we must find a way of introducing only the curl-free contribution from the electric field estimate $w \mathbf{E}^{\mathrm{PIL}}$. This can be accomplished if we first write the resulting electric field in terms of a scalar potential, $\psi^{D}$, such that

$$
c \mathbf{E}_{h}^{D}=-\nabla_{h} \psi^{D},
$$

and then set the divergence of $c \mathbf{E}^{D}$ to the divergence of $c w \mathbf{E}^{\mathrm{PIL}}$, resulting in the Poisson equation

$$
\nabla_{h}^{2} \psi^{D}=-c \nabla_{h} \cdot\left(w \mathbf{E}^{\mathrm{PIL}}\right)
$$

that we solve for $\psi^{D}$ using FISHPACK. Since $\mathbf{E}_{h}^{D}$ is the gradient of a scalar function, it automatically has zero curl.

When $\mathbf{E}_{h}^{D}$ derived from $\psi^{D}$ is added to the PTD solution $\mathbf{E}^{P}$, we denote the resulting solution as the PD electric field. If we then add the ideal correction $\mathbf{E}^{I}$ to the PD solution, by applying the $\mathbf{E} \cdot \mathbf{B}=0$ constraint, we denote that resulting electric field as the PDI solution.

\subsubsection{FLCT-derived Electric Fields}

Local Correlation Tracking (LCT) techniques have been used extensively in recent years to estimate flows in the solar atmosphere, as noted in the introduction. These methods use observed pattern motions of some quantity such as the specific intensity or the normal component of the magnetic field to provide an estimate of the velocity components of the plasma that are parallel to the plane of the image. The input to LCT techniques is the following: two images of the quantity that is being tracked, the time difference between the two images, and an a priori assumption about the coherence length of the flowfield. The output from LCT is a two-dimensional flow field that warps the first image into the second image over the elapsed time between the images. The fundamental assumption of LCT techniques is that the observed pattern motion corresponds to real physical motion. In the remainder of this paper, we will focus on the use of the Fourier Local Correlation Tracking (FLCT) technique (Welsch et al. 2004; Fisher \& Welsch 2008), but any other optical flow method (Schuck 2006) could be used instead.

When the quantity being tracked is the normal component of the magnetic field, velocity estimates obtained from LCT methods have been found to provide a good estimate on their own for the "inductive" flows responsible for changes in the normal field (see, e.g., Figure 6 of Welsch et al. 2007). However, LCT results also contain information about flows that do not contribute directly to the observed changes in the magnetic field tracer, and thus provide additional information about the velocity field (or the electric field, assuming an ideal Ohm's law). The ILCT method (Welsch et al. 2004) uses this knowledge to explicitly separate the flow field into inductive and non-inductive contributions.

In our case, $\mathbf{E}^{P}$ already contains the "inductive" contribution to the electric field, so a straightforward addition of the electric field from flows derived from FLCT would add redundant information that has already been incorporated while also adding new information from the non-inductive flows. We therefore want to determine only the non-inductive contribution to the electric field from FLCT-derived flows and add that result to $\mathbf{E}^{P}$. The technique for doing this is very similar to how we add the non-inductive electric field determined from the Doppler measurements.
Assuming the ideal Ohm's law, we can write the electric field derived from FLCT as

$$
c \mathbf{E}=-\left(\mathbf{V}_{h} \times B_{z} \hat{\mathbf{z}}\right)-\left(\mathbf{V}_{h} \times \mathbf{B}_{h}\right),
$$

where $\mathbf{V}_{h}$ here is the horizontal flow-field from FLCT. This results in a contribution to both $\mathbf{E}_{h}$ (the first term in Equation (25)), and $E_{z}$, the second term in that equation. Considering first the contribution of FLCT to $\mathbf{E}_{h}$, we note that as one approaches the PIL, this estimate for $\mathbf{E}_{h}$ becomes increasingly suspect since $B_{z}$, the quantity being tracked, becomes very small, and the validity of the correlation tracking paradigm to describe the evolution of $B_{z}$ becomes increasingly questionable. Since the FLCT results get unreliable precisely where we think the Doppler electric field estimate becomes reliable, we make the Ansatz that the FLCT-derived electric field should be multiplied by the complement $(1-w)$ of the same confidence factor, $w$, used to modulate the Doppler electric field.

To include only the non-inductive contribution, we write the electric field in terms of the gradient of a scalar potential,

$$
c \mathbf{E}_{h}^{\mathrm{FLCT}}=-\nabla_{h} \psi^{\mathrm{FLCT}} .
$$

This electric field has no curl and hence no inductive contribution. To determine $\psi^{\text {FLCT }}$, we multiply the right-hand side of Equation (25) by $(1-w)$, take its horizontal divergence, and then set that equal to the divergence of Equation (26):

$$
\nabla_{h}^{2} \psi^{\mathrm{FLCT}}=\nabla_{h} \cdot(1-w) \mathbf{V}_{h} \times B_{z} \hat{\mathbf{z}}
$$

The result of this solution is an electric field that includes the non-inductive contributions from FLCT, which can then be added to $\mathbf{E}_{h}^{P}$. This procedure is very similar to ILCT (Welsch et al. 2004), apart from (1) using electric fields instead of flowfields and (2) the use of the confidence factor.

Now, we consider the contributions to $E_{z}$ from the second term in Equation (25), and how to correct this for the inductive contribution to $E_{z}$ from $\mathbf{E}^{P}$. First, note that the FLCT estimate for $E_{z}$ is approximately correct, but has a lot of scatter (see, e.g., the second panel of Figure 11 in Welsch et al. 2007). Our own comparison of $E_{z}$ from FLCT with that from $E_{z}^{P}$ shows that the FLCT contribution contains significant information that overlaps with $E_{z}^{P}$. Since the inductive contribution is already completely contained within $c E_{z}^{P}=-\dot{\mathcal{J}}$, we need to remove any inductive signature from the FLCT estimate for $E_{z}$. The result of doing this is a residual contribution to $E_{z}$ that satisfies the horizontal Laplace equation but obeys the Dirichlet boundary conditions for $E_{z}$ that are returned from FLCT. If the boundary electric fields are near zero, as we generally desire, then the resulting FLCT residual $E_{z}$ contribution is generally small, since solutions of Laplace's equation achieve their extremum values at the boundaries. The residual $E_{z}$ contribution is certainly very small in the validation test case considered later in this paper as well as for our planned HMI inversions, and we therefore do not include it in our inversion estimates at this time.

When the $\mathbf{E}_{h}^{\mathrm{FLCT}}$ solution is added to the PTD electric field $\mathbf{E}^{P}$, we denote the resulting electric field as the PF solution. If the ideal electric field is added to that solution to impose the constraint $\mathbf{E} \cdot \mathbf{B}=0$, we denote the resulting electric field as the PFI solution. If the FLCT electric field and the Doppler electric field are added to $\mathbf{E}^{P}$, we denote the resulting electric field solution as the PDF solution. Finally, if we impose the ideal electric field constraint to the PDF solution, we obtain what 
Table 1

Summary of Electric Field Notation

\begin{tabular}{lccc}
\hline \hline Name & Denoted & Equation for $\mathbf{E}$ & Input Data / Constraints \\
\hline P solution $^{\mathrm{a}}$ & $\mathbf{E}^{\mathbf{P}}$ & $c \mathbf{E}=c \mathbf{E}^{\mathbf{P}}$ & $\dot{B}_{z}, \dot{J}_{z}($ generally, $\mathbf{E} \cdot \mathbf{B} \neq 0)$ \\
PI solution $^{\mathrm{b}}$ & $\mathbf{E}^{\mathrm{PI}}$ & $c \mathbf{E}=c \mathbf{E}^{\mathbf{P}}-\nabla \psi^{I}$ & $\dot{B}_{z}, \dot{J}_{z}, \mathbf{E} \cdot \mathbf{B}=0$ \\
PFI solution $^{\mathrm{c}}$ & $\mathbf{E}^{\mathrm{PFI}}$ & $c \mathbf{E}=c \mathbf{E}^{\mathbf{P}}+c \mathbf{E}_{\mathbf{t}}^{\mathrm{FLCT}}-\nabla \psi^{I}$ & $\dot{B}_{z}, \dot{J}_{z}, \mathbf{E} \cdot \mathbf{B}=0$, FLCT output \\
PDI solution $^{\mathrm{d}}$ & $\mathbf{E}^{\mathrm{PDI}}$ & $c \mathbf{E}=c \mathbf{E}^{\mathbf{P}}+c \mathbf{E}^{\mathbf{D}}-\nabla \psi^{I}$ & $\dot{B}_{z}, \dot{J}_{z}, \mathbf{E} \cdot \mathbf{B}=0$, Dopp. data \\
PDFI solution & $\mathbf{E}^{\mathrm{PDFI}}$ & $c \mathbf{E}=c \mathbf{E}^{\mathbf{P}}+c \mathbf{E}^{\mathbf{D}}+c \mathbf{E}_{\mathbf{t}}^{\mathrm{FLCT}}-\nabla \psi^{I}$ & $\dot{B}_{z}, \dot{J}_{z}, \mathbf{E} \cdot \mathbf{B}=0$, Dopp. \& FLCT \\
\hline FI solution & $\mathbf{E}^{\mathbf{F I}}$ & $c \mathbf{E}=-\mathbf{V}_{\mathbf{h}} \times \mathbf{B}^{\mathrm{e}}$ & FLCT data, $V_{\text {LOS }}=0$ \\
DI solution & $\mathbf{E}^{\text {DI }}$ & $c \mathbf{E}=-\mathbf{V}_{\mathrm{LOS}} \times \mathbf{B}^{\mathrm{e}}$ & Dopp. data, $V_{h}=0$ \\
DFI solution & $\mathbf{E}^{\mathrm{DFI}}$ & $c \mathbf{E}=-\mathbf{V} \times \mathbf{B}^{\mathrm{e}}$ & Dopp. \& FLCT data \\
\hline
\end{tabular}

Note.

a $\mathrm{P}$ for PTD.

b I for ideal.

${ }^{c} \mathrm{~F}$ for FLCT.

${ }^{\mathrm{d}} \mathrm{D}$ for Doppler.

${ }^{\text {e }}$ For FI, DI, and DFI, we do not include $\nabla \psi^{I}$ because the total electric field is already ideal.

we denote as the PDFI solution. As discussed in greater detail later in this article, we generally find that the PDFI solution provides the best results when compared to the electric fields in our standard test case.

\subsubsection{Doppler Electric Fields: Non-normal Viewing Angles}

Viewing the solar surface from a non-normal angle $(\theta \neq 0)$ introduces some complications for estimating the electric field. These are due to (1) foreshortening effects and (2) changes in the direction of the flows derived from Doppler measurements. Because our assumed magnetic field data is from vector magnetograms, we can correct it for foreshortening, interpolate it to a uniform grid on the solar surface, and reproject the magnetic field directions into the locally normal and horizontal components. This means that the PTD inversion technique for $\mathbf{E}^{P}$ can be applied to the remapped data without any change in the method, as can FLCT, to derive part of the non-inductive the electric field. Using the Doppler velocity to compute noninductive electric fields along the solar surface, however, becomes more difficult and some additional steps must be taken.

We first note that if the angle of the observation is written in terms of the unit line-of-sight (LOS) vector $\hat{\mathbf{l}}=$ $[\sin (\theta), 0, \cos (\theta)]$, where the direction of this vector is toward the observer as measured from the solar surface, then the velocity vector defined by the Doppler shift is given by

$$
\mathbf{V}_{\mathrm{LOS}}=(\mathbf{V} \cdot \hat{\mathbf{l}}) \hat{\mathbf{l}},
$$

where $\mathbf{V}$ is the plasma velocity at the source point on the solar surface. The geometry is shown in Figure 1.

The electric field due to the Doppler velocity $\mathbf{V}_{\text {LOS }}$ near LOS PILs is then given by

$$
c \mathbf{E}=-\mathbf{V}_{\mathrm{LOS}} \times \mathbf{B}_{t},
$$

where here $\mathbf{B}_{t}$ represents the components of $\mathbf{B}$ in the directions transverse to the LOS direction. Note that this electric field is no longer confined to directions parallel to the solar surface, but instead is confined to the directions normal to î. Similar to the normal-viewing angle case, we anticipate that as the ratio $\left|B_{\mathrm{LOS}}\right| /\left|B_{t}\right|$ increases above zero, our confidence in this electric field to represent the transverse electric field decreases due to possible parallel flows and other contributions to the transverse electric field. Therefore we multiply $\mathbf{E}$ by an ad hoc confidence factor

$$
w_{\mathrm{LOS}}=\exp \left[-\frac{1}{\sigma_{\mathrm{PIL}}^{2}}\left|\frac{B_{\mathrm{LOS}}}{B_{t}}\right|^{2}\right],
$$

where $\sigma_{\mathrm{PIL}}$ has the same value as $\sigma_{\mathrm{PIL}}$ in Equation (22) for $w$. Note that the confidence factor used for determining $E^{\mathrm{FLCT}}$ is $1-w$ and not $1-w_{\text {LOS }}$, since the FLCT formalism is not affected by non-normal viewing angles.

To determine non-inductive contributions from the Doppler electric field, we borrow the iterative technique from Section 2.2, and find a scalar potential function $\psi^{D}$ that obeys the constraint

$$
\nabla \psi^{D} \cdot \hat{\mathbf{q}}=w_{\mathrm{LOS}} \mathbf{V}_{\mathrm{LOS}} \times \mathbf{B}_{t} \cdot \hat{\mathbf{q}},
$$

where $\hat{\mathbf{q}}$ is the unit vector pointing in the direction of $\mathbf{V}_{\mathrm{LOS}} \times \mathbf{B}_{t}$. Note the analogy between the roles of $\psi^{D}$ and $\hat{\mathbf{q}}$ in Equation (31) and $\psi^{I}$ and $\hat{\mathbf{b}}$ in Equation (18). Accordingly, as in Equation (19), we then decompose Equation (31) into the directions parallel and perpendicular to the solar surface:

$$
\begin{aligned}
\nabla_{h} \psi^{D} \cdot \hat{\mathbf{q}}_{h}+q_{z} \partial \psi^{D} / \partial z= & w_{\mathrm{LOS}}\left(\mathbf{V}_{\mathrm{LOS}} \times \mathbf{B}_{t}\right)_{h} \cdot \hat{\mathbf{q}}_{h} \\
& +q_{z} w_{\mathrm{LOS}}\left(\mathbf{V}_{\mathrm{LOS}} \times \mathbf{B}_{t}\right)_{z}
\end{aligned}
$$

where $\hat{\mathbf{q}}_{h}$ and $q_{z}$ represent the horizontal and vertical components of $\hat{\mathbf{q}}$, respectively. We then solve Equation (32) for $\psi^{D}$ and $\partial \psi^{D} / \partial z$ using exactly the same "iterative" procedure described in Section 2.2. The result is a curl-free electric field that can then be added to $\mathbf{E}^{P}$. Henceforth, even if the viewing angle is normal to the solar surface, we use the non-normal formalism for computing electric fields from Doppler shifts but then set $\theta=0$ in expression for $\hat{l}$.

\subsubsection{Combinations of Electric Field Contributions: A Summary}

Now that we have introduced the different possible electric field contributions, we summarize the naming convention we use henceforth in this article, depending on which contributions are included in an electric-field estimate (see Table 1). To distinguish between different versions of electric field inversions we use combinations of the following four letters: "P" (for PTD, inductive), "D" (for Doppler, non-inductive), "F" (for FLCT, non-inductive), and "I" (for ideal, non-inductive). Solutions with an "I" in their name have the ideal-MHD constraint, 
i.e., the addition of the field from the potential $-\nabla \psi^{I}$, imposed as the last step. We also consider the full FLCT results, denoted FI, the full Doppler electric field results, denoted DI, and their sum, denoted DFI. For FI, DI, and DFI, we did not impose $\nabla \psi^{I}$ because their total electric field is already ideal. Consequently, we have a notation for the electric field, helicity and Poynting fluxes computed with a variety of different electric field estimation techniques.

The biggest changes from the solutions described here and those of Fisher et al. (2012) are the adoption of the FISHPACK software to solve the two-dimensional Poisson equations, the ability to compute contributions to Doppler-shift electric fields from non-normal viewing angles, and a much more systematic and quantitative testing of the accuracy and robustness of the technique (described further in Section 4).

\section{USING ELECTRIC FIELDS TO COMPUTE POYNTING AND HELICITY FLUXES}

\subsection{Poynting Flux Components: $S_{z \text {,pot }}$ and $S_{z \text {,free }}$}

The Poynting-flux vector

$$
\mathbf{S}=\frac{c}{4 \pi}(\mathbf{E} \times \mathbf{B})
$$

measures the flow of electromagnetic energy at the photosphere, where magnetic field vector $\mathbf{B}$ is defined from the observations, and the electric field vector $\mathbf{E}$ is taken from the techniques described in Section 2. Since we are interested in the amount of energy flowing into and out of the corona, we focus most of our attention on the vertical component of Poynting flux, $S_{z}$. This depends upon the horizontal components of both the electric field and the magnetic field:

$$
S_{z}=\frac{c}{4 \pi}\left(E_{x} B_{y}-E_{y} B_{x}\right)=\frac{c}{4 \pi}\left(\mathbf{E}_{h} \times \mathbf{B}_{h}\right) \cdot \hat{\mathbf{z}} .
$$

Computing $S_{z}$ is straightforward, given our techniques for finding all three components of $\mathbf{E}$ as described above and the availability of the components of $\mathbf{B}$ from the vector magnetogram data. We can go further and decompose $S_{z}$ into two contributions, the flux of potential-field energy and the flux of free magnetic energy. The distinction between these two contributions was first made by Welsch (2006), who expressed the Poynting flux in terms of velocities. An expression for the flux of free magnetic energy was given in Fisher et al. (2010) in terms of the electric field and a decomposition of the horizontal magnetic field in terms of the poloidal and toroidal potentials. The basic idea is that the horizontal magnetic field $\mathbf{B}_{h}$ can be divided into a potential-field contribution and a contribution due to currents that flow into the atmosphere from the photosphere. Because there are several ways that one can construct a potential field from the photospheric vector magnetogram data, we first discuss what we believe is the best way of performing this decomposition and then briefly mention another alternative we have considered.

Horizontal electric fields derived from Faraday's law are derived, in part, from the observed evolution of the vertical magnetic field. Therefore, the most self-consistent description of the potential magnetic field is to find the potential field that best matches the values of the measured normal component of the magnetic field at the photosphere. There are a number of ways to do this using, e.g., Green's function techniques or FFTs (though in the latter case, one must ensure the region is flux balanced and that sufficient padding is provided outside of the active region to mitigate against artifacts from periodic boundary conditions). Once the potential function $\Phi$ has been derived, the horizontal potential magnetic field at the photosphere can be evaluated as

$$
\mathbf{B}_{h}^{P}=-\nabla_{h} \Phi \text {. }
$$

The flux of potential-field energy is then given by

$$
S_{z, \mathrm{pot}}=\frac{1}{4 \pi} c \mathbf{E}_{h} \times \mathbf{B}_{h}^{P}
$$

To compute the flux of free magnetic energy, we use the residual horizontal field that is the difference between the measured and potential components:

$$
S_{z, \text { free }}=\frac{1}{4 \pi} c \mathbf{E}_{h} \times\left(\mathbf{B}_{h}-\mathbf{B}_{h}^{P}\right) .
$$

We have also experimented with using the potential field formulation suggested in Appendix A of Fisher et al. (2010). This has the advantage of being extremely easy to compute in terms of the poloidal-toroidal potential function $\partial \mathcal{B} / \partial z$, which has the same PTD formalism we use to solve for $\mathbf{E}^{P}$. However, as B. T. Welsch \& G. H. Fisher (2014, in preparation) have shown, this potential field matches the observed values of $\nabla_{h} \cdot \mathbf{B}_{h}$ at the photosphere, and may not accurately reflect the measured value of $B_{z}$. If the actual magnetic field were in fact potential, B. T. Welsch \& G. H. Fisher (2014, in preparation) show that the two formulations would provide the same result. Since currents are present, however, the two formulations can and do show differences in $B_{z}$ at the photosphere. Since our derived electric fields are driven primarily by changes in $B_{z}$, we therefore choose a potential field that explicitly matches $B_{z}$ at the photosphere.

For short sequences of vector magnetograms, like, e.g., the analysis of the ANMHD test data described in Section 4, we compute the potential magnetic field by using the Green's function technique (see Appendix B of B. T. Welsch \& G. H. Fisher 2014, in preparation), as we have found it to be the most robust and accurate. It is, however, very computationally intensive and can be impractical for large data sets. For long cadences of large vector magnetograms, we use a padded FFT solution for the potential magnetic field with the size of the needed padding area calibrated by solutions from the Green's function technique.

\subsection{Helicity Flux Rate: $d H_{R} / d t$}

The magnetic helicity of magnetic field $\mathbf{B}$ in a volume $V$

$$
H=\int \mathbf{A} \cdot \mathbf{B} d V,
$$

where

$$
\mathbf{B}=\nabla \times \mathbf{A}
$$

measures the linkage of magnetic field lines and is useful for describing their topology. Magnetic helicity is physically meaningful only for magnetic fields that are fully contained in a volume $V$, a condition which for active regions with magnetic field lines extending far above and below the photosphere, is not satisfied. In this case, subtracting the helicity of the potential field $\mathbf{B}_{\mathbf{p}}$ that matches the normal component of $B_{n}$ on $S$, one can define topologically meaningful and gauge-invariant relative magnetic helicity, i.e., helicity relative to the potential field (Berger \& Field 1984; Finn \& Antonsen 1985)

$$
H_{R}=\int_{V}\left(\mathbf{A}+\mathbf{A}_{\mathbf{p}}\right) \cdot\left(\mathbf{B}-\mathbf{B}_{\mathbf{p}}\right) d V .
$$


Due to absence of accurate magnetic field measurements throughout the corona, it is hard to derive relative magnetic helicity directly from the observations. Instead, rate of change of relative helicity, $d H_{R} / d t$ is used to characterize the coronal magnetic field complexity. If we know the electric field, then the time rate of change of relative helicity in volume $V$-equivalent to the flux of helicity into $V$-is given by the surface integral (Equation (62) in Berger \& Field 1984)

$$
\begin{aligned}
\left(\frac{d H_{R}}{d t}\right) & =-2 \int\left(\mathbf{A}_{\mathbf{p}} \times \mathbf{E}\right) \cdot \hat{\mathbf{z}} d a \\
& =-2 \int\left(A_{p x} E_{y}-A_{p y} E_{z}\right) d a
\end{aligned}
$$

where $\mathbf{A}_{\mathbf{p}}=((\partial \mathcal{B}) /(\partial y),-(\partial \mathcal{B}) /(\partial x), 0)=\nabla \times \mathcal{B} \hat{\mathbf{z}}$ is the vector potential that generates the potential field $\mathbf{B}_{\mathbf{p}}$ in $V$, which matches the photospheric normal field $B_{z}$. We require that $\mathbf{A}_{\mathbf{p}}$ obey the Coulomb gauge condition (divergence-free in $V$ ) and be tangential on the bounding surface. For a closed magnetic field rooted in the photosphere, the helicity flow across the upper boundaries is zero and only photospheric surface term remains.

If we have an ideal electric field $c \mathbf{E}=-\mathbf{V} \times \mathbf{B}$, then the helicity flux rate, given by Equation (41), splits into two terms (Berger 1984):

$$
\left(\frac{d H_{R}}{d t}\right)=\underbrace{-2 \int\left(\mathbf{A}_{\mathbf{p}} \cdot \mathbf{V}_{\mathbf{h}}\right) B_{z} d a}_{\text {braiding, FI }}+\underbrace{2 \int\left(\mathbf{A}_{\mathbf{p}} \cdot \mathbf{B}_{\mathbf{h}}\right) V_{z} d a}_{\text {emergence, DI }} .
$$

The first term corresponds to magnetic helicity generated by flux tubes moving horizontally in the photosphere (braiding), and the second term corresponds to helicity injection due to emergence from the solar interior into the corona. Note that adopting the naming convention from Section 2.3.4, braiding and emergence terms would correspond to the FI and DI electricfield solutions, respectively, and their sum to the DFI solution. When using the electric field form of the relative helicity flux (Equation (41)), the braiding and emergence terms are not neatly separated.

In Section 4, using ANMHD test data with a known helicity flux rate, we use both the electric field (Equation (41), PDFI-, PDI-, or PFI-solution) and the velocity field (Equation (42), FI-, DI-, or DFI-solution) to evaluate the best approach for estimating $d H_{R} / d t$.

\section{VALIDATION OF THE ELECTRIC FIELD INVERSION METHOD USING ANMHD SIMULATIONS}

\subsection{ANMHD Run: Input, Output, Parameters}

In this paper, we use a specific set of anelastic pseudo-spectral ANMHD simulations (Fan et al. 1999; Abbett et al. 2000, 2004) of an emerging magnetic bipole in a convecting box (Welsch et al. 2007) to test our improved electric field inversion technique. From ANMHD magnetic fields and plasma velocities, we know the actual electric fields, which we can compare to electric fields derived using the simulation's evolving magnetic field. In the past, this ANMHD simulation has been used for several studies of velocity field inversions (Welsch et al. 2007; Schuck 2008) as well as in test cases for the first electric field inversions (Fisher et al. 2010, 2012). Fisher et al. (2012) showed that the PDFI solution significantly improves the accuracy of the derived PI solution, and the improvement from the knowledge of the Doppler velocity (PDI) is significantly more important than that of the horizontal velocity (PFI), at least in this example of magnetic flux emergence. In this paper, we perform a series of improvements to PDFI method beyond Fisher et al. (2012): we expand the derivation of the non-inductive contribution to nonnormal viewing angles, introduce spherical coordinates for the PTD solution (see the Appendix), and significantly speed up the Poisson equation solutions by using FISHPACK. With the above upgrades, we find that the PDFI method yields a good estimate of the electric field, Poynting, and helicity fluxes and is ready to be applied routinely to the observed vector magnetograms.

We use a pair of ANMHD vector magnetograms, separated by $\Delta t=250 \mathrm{~s}$ with a pixel size of $\Delta s=348.36 \mathrm{~km}$, and a LOS velocity map, observed at a specific viewing angle, to derive the electric field and the vertical Poynting flux for a given set of parameters (see Table 2). To estimate the horizontal velocity field, $\left(V_{x}, V_{y}\right)$, we use the Fourier localcorrelation tracking (FLCT) technique (Welsch et al. 2004; Fisher \& Welsch 2008) (http://solarmuri.ssl.berkeley.edu/ fisher/ public/software/FLCT/C_VERSIONS/). We denote the Gaussian window size scale (a parameter in FLCT) by $\sigma_{\text {FLCT }}$. To suppress noisy behavior, we only calculate velocities where $\left|B_{z}\right|>$ $370 \mathrm{G}$, or $5 \%$ of maximum $\left|B_{z}\right|$ (Welsch et al. 2007).

In Table 2 we summarize all the input and output variables of the PDFI run and their typical ranges. Further, we vary the observed viewing angle $\theta$ within $[0,60]^{\circ}$ range to estimate the accuracy of the PDFI method at non-zero viewing angles. We also vary the other free parameters: the number of iterations in the perpendicularization technique ( $N_{\text {iter }}$; see Section 2.2), the width of the Gaussian window used in the FLCT estimate for the horizontal velocity $\left(\sigma_{\mathrm{FLCT}}\right.$; see below $)$, and the width of polarity inversion line ( $\sigma_{\mathrm{PIL}}$; see Section 2.3.1) to find the best values of the parameter set that yields the most accurate electric field solution.

For the remainder of this paper, to assess the performance of our methods, i.e., of the reconstruction $u^{\prime}$ of the ANMHD variable $u$, we use the following metrics: (1) a fraction of the integrated total $f\left(u, u^{\prime}\right)=\sum_{i} u^{\prime} / \sum_{i} u$; (2) the slope or linear coefficient in the least-squares, polynomial fit, $a\left(u, u^{\prime}\right): u^{\prime} \approx$ $a_{0}+a\left(u, u^{\prime}\right) * u$; (3) the linear Pearson correlation coefficient $\rho\left(u, u^{\prime}\right)=\left(\operatorname{cov}\left(u, u^{\prime}\right)\right) /\left(\sigma_{u} \sigma_{u^{\prime}}\right)$, where $\sigma_{u}$ is a standard deviation of $u$; and (4) the normalized error of $u^{\prime}, E r r .=\sigma\left(u^{\prime}-u\right) / \sigma_{u}$. $E r r .=0.1$ means that the error of the reconstruction is $10 \%$ relative to the characteristic range of $u$. Note that to exclude the weak-field background of $u$ where the observed magnetic field (e.g., in HMI/SDO) tends to be noisy, we estimated $f, a, \rho$ and err. in locations where $|B|=\left(B_{x}{ }^{2}+B_{y}{ }^{2}+B_{z}{ }^{2}\right)^{1 / 2}>370 \mathrm{G}$. In the HMI vector magnetogram case, a similar threshold will be determined from estimated errors in the magnetic field values. To summarize, the ideal reconstruction $u^{\prime}$ of variable $u$ satisfies: $f\left(u, u^{\prime}\right)=1, a\left(u, u^{\prime}\right)=1, \rho\left(u, u^{\prime}\right)=1$ and Err. $=0.0$.

Figure 2 shows three panels that quantitatively justify selection of the best set of PDFI parameters (vertical dotted lines): number of iterations $\left(N_{\text {iter }}\right.$, left panel), width of the Gaussian window $\left(\sigma_{\mathrm{FLCT}}\right.$, middle panel $)$, and the PIL width $\left(\sigma_{\mathrm{PIL}}\right.$, right panel). The left panel, $\alpha_{E B}\left(N_{\text {iter }}\right)$, shows dependence of the rms angle between the electric and magnetic field vectors on the number of iterations using the perpendicularization technique (see Section 2.2). When looking for the optimal number of iterations $N_{\text {iter }}$, we try to keep $N_{\text {iter }}$ as low as possible to achieve a high-speed performance while still aiming for an angle close to $90^{\circ}$. For the ANMHD case, without 

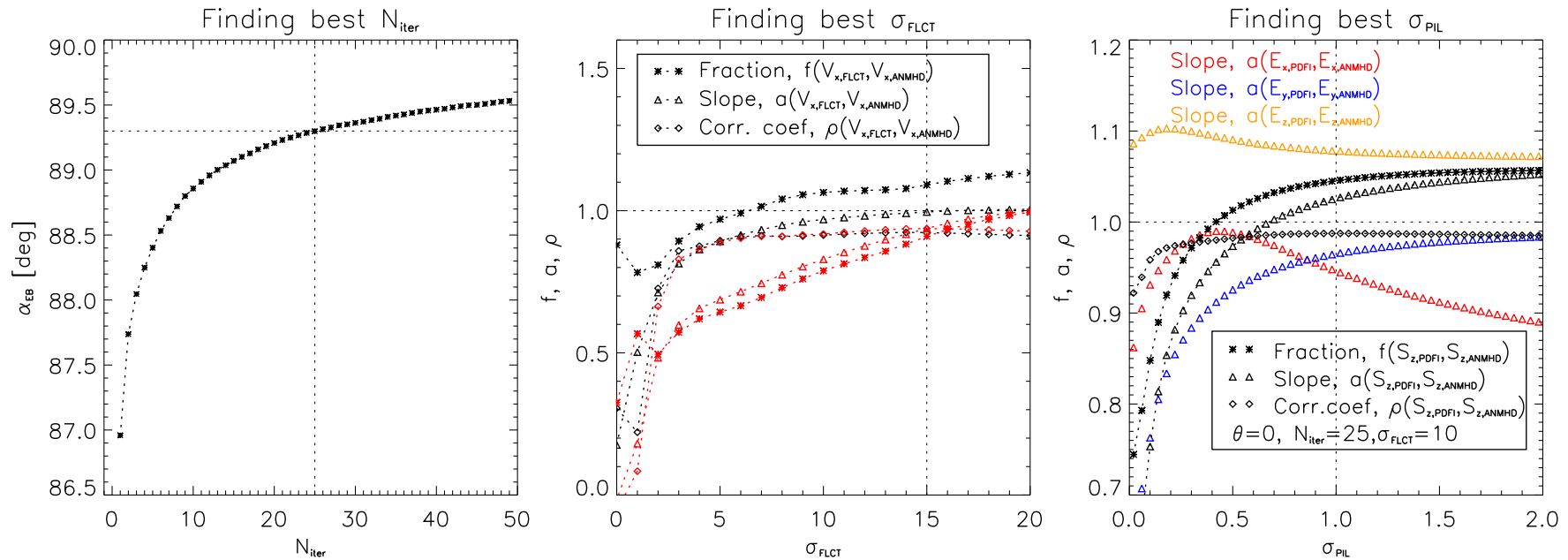

Figure 2. Finding best set of parameters for PDFI: $N_{\mathrm{iter}}, \sigma_{\mathrm{FLCT}}$ and $\sigma_{\mathrm{PIL}}$. Left: dependence of the angle between $\mathrm{E}$ and B on the number of steps in the perpendicularization process, $\alpha_{E B}\left(N_{\text {iter }}\right)$. Middle: quality $(f, a, \rho)$ of horizontal velocity components $V_{x}$ (black) and $V_{y}$ (red), reconstructed using FLCT technique at different values of the Gaussian window width $\sigma_{\mathrm{FLCT}}$. Right: black curves show the quality $(f, a, \rho)$ of the $S_{z}$ reconstruction at different PIL widths $\sigma_{\mathrm{PIL}}$. Red, blue, and yellow colors show the slopes $a\left(E_{x, \mathrm{PDFI}}, E_{x, \mathrm{ANMHD}}\right), a\left(E_{y, \mathrm{PDFI}}, E_{y, \mathrm{ANMHD}}\right)$, and $a\left(E_{z, \mathrm{PDFI}}, E_{z, \mathrm{ANMHD}}\right)$, respectively, they quantify the faithfulness of the $E_{x}, E_{y}$, and $E_{z}$ reconstructions at different values of $\sigma_{\mathrm{PIL}}$. Vertical dotted lines show the best values of parameters that we further use as default.

(A color version of this figure is available in the online journal.)

Table 2

Input and Output Variables of a Set of PDFI Runs for the ANMHD Test Case and their Typical Range

\begin{tabular}{lcc}
\hline \hline Input & Description & Observed Range \\
\hline$B_{t, x}, B_{t, y}, B_{\mathrm{LOS}}(x, y)$ & Magnetic field & {$[-6000,6000] \mathrm{Gauss}$} \\
$V_{\mathrm{LOS}}(x, y)$ & Doppler velocity & {$[-0.5,0.2] \mathrm{km} \mathrm{s}^{-1}$} \\
$\theta$ & Viewing angle & {$[0,60]^{\circ}$} \\
\hline Output & & \\
$V_{x}, V_{y}(x, y)$ & FLCT velocity field & {$[-0.4,0.4] \mathrm{km} \mathrm{s}^{-1}$} \\
$E_{x}, E_{y}, E_{z}(x, y)$ & PDFI electric field & {$[-1,1] \mathrm{cm}^{-1}$} \\
$S_{z}(x, y)$ & Poynting flux & {$[-2,6] \times 10^{10} \mathrm{ergs}^{-} /\left(\mathrm{s} \mathrm{cm}^{2}\right)$} \\
\hline Parameters & & Range (Best value) \\
$N_{\text {iter }}$ & No. of iterations in $\mathbf{E} \cdot \mathbf{B}=0$ & {$[0,50]$ iterations $(25)$} \\
$\sigma_{\mathrm{FLCT}}$ & Gaussian window width & {$[0,15]$ pixels $(15)$} \\
$\sigma_{\mathrm{PIL}}$ & PIL width & {$[0,2]$ pixels $(1)$} \\
\hline
\end{tabular}

Notes. To test the PDFI method, we varied the parameters within a shown range. We then used the best-value parameter shown in the parenthesis as a default.

perpendicularization $\left(N_{\text {iter }}=0\right)$, the angle between the magnetic and electric field vectors $\alpha_{E B}=75^{\circ}$. After only one iteration $\left(N_{\text {iter }}=1\right), \alpha_{E B}=87^{\circ}$. The angle slowly increases to $\alpha_{E B}=89.3$ by $N_{\text {iter }}=25$. The convergence rate slows down, and reaches $\alpha_{E B}=89.5$ by $N_{\text {iter }}=50$. We chose $N_{\text {iter }}=25$ as the optimal number of iterations, since above 25 the convergence of $\alpha_{E B}$ toward $90^{\circ}$ is too slow to justify the additional computational effort. We also use $N_{\text {iter }}=25$ when applying the non-normal viewing angle technique (Section 2.3.3) for finding the needed scalar potential $\psi^{D}$.

The middle panel of Figure 2 shows how the quality of the horizontal velocity reconstruction, using the FLCT technique in the ANMHD test case, depends on the Gaussian window width $\sigma_{\text {FLCT }}$. Since velocities parallel to the magnetic field do not affect the time evolution of the magnetic field and hence the fluxes of magnetic energy and helicity, in this plot we only compare components of the flow field that are perpendicular to $\mathbf{B}$. We find that $\sigma_{\mathrm{FLCT}}<6$ pixels yields the worst agreement with the actual horizontal ANMHD plasma speed; $\sigma_{\mathrm{FLCT}}=15$ pixels yields the best agreement and we therefore adopt it as the default value.
For comparison, in Welsch et al. (2007), the optimal Gaussian window size was also chosen to be $\sigma_{\mathrm{FLCT}}=15$ and in Fisher et al. (2012) $\sigma_{\text {FLCT }}=5$.

Finally, the right panel of Figure 2, "Finding the best

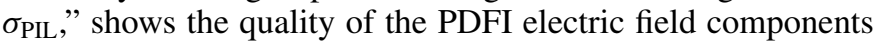
(red, blue, orange) and Poynting flux (black) reconstruction for different $\sigma_{\mathrm{PIL}}$ 's, a free parameter that reflects the lack of confidence in the accuracy of the horizontal Doppler electric field away from PILs (see Section 2.3). The panel shows that $\sigma_{\mathrm{PIL}}$ has a small effect on the quality of $E_{y}, E_{z}$ and $S_{z}$, above $\sigma_{\mathrm{PIL}}=1$. We find that $\sigma_{\mathrm{PIL}} \simeq 1$ yields the best ratio between the total reconstructed and ANMHD Poynting fluxes: $\rho \simeq 1, a \simeq 1$.

Using the best set of parameters $\left(N_{\text {iter }}=25, \sigma_{\mathrm{FLCT}}=15\right.$ and $\sigma_{\mathrm{PIL}}=1$ ) found in Section 4.2, we estimate the quality of the PDFI reconstructed electric fields, helicity and Poynting fluxes and also estimate the uncertainties in the results at a zero viewing angle. In Section 4.3, we describe how observing at the non-zero viewing angles affects the quality of the reconstruction. Finally in Section 4.4, we estimate the roles that different non-inductive 

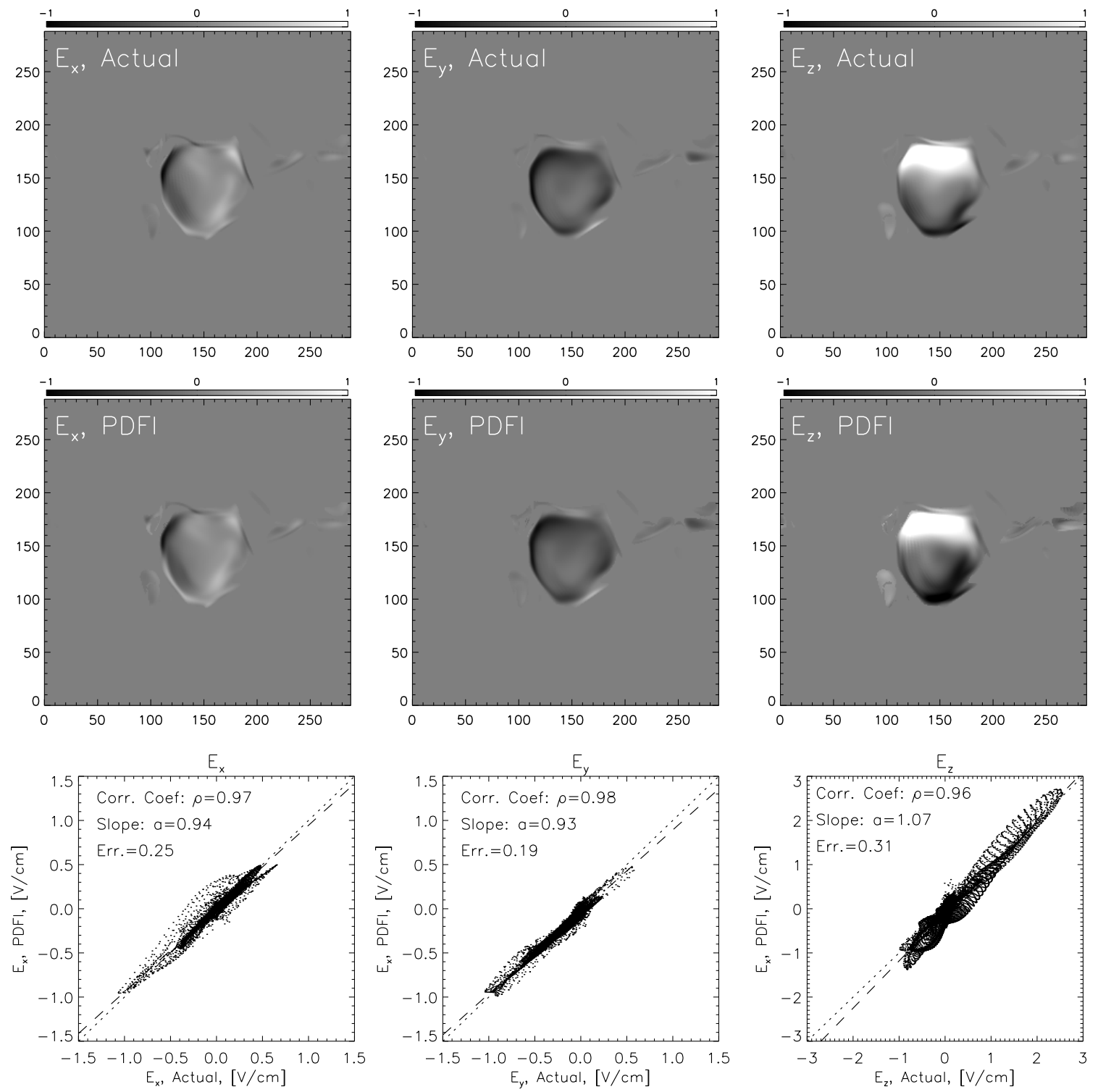

Figure 3. Validation of the PDFI electric field at $\theta=0^{\circ}$ : actual (top row) and PDFI (middle row) electric field vector components, [ $\left.E_{x}, E_{y}, E_{z}\right]$, (left, middle, right) for the ANMHD test case. Bottom row: pixel-by-pixel scatter plots comparing the top two rows. The slopes of the linear fits and correlation coefficients are given in the top left corners.

Doppler and FLCT contributions play in the reconstruction and compare current results to Fisher et al. (2012).

\subsection{Results: Electric Field, Poynting and Helicity Fluxes}

Figures 3 and 4 show validation plots that compare electric field components $\left(E_{x}, E_{y}, E_{z}\right)$ and vertical Poynting fluxes derived from the PDFI-method with the actual ANMHD quantities. The PDFI-method reconstructs the ANMHD electric-field components quite well: the top and middle rows of Figures 3 look almost identical. The slope of the linear fit to the reconstructed versus the actual electric-field component ranges from $a=0.94$ to $a=1.07$. The correlation coefficient, describing the quality of linear fit, is close to one in all cases. Using these electric-field components, we also find a good agreement for the vertical Poynting flux (Figure 4): the correlation coefficient $\rho=0.99$, the slope $a=0.98$, and the fraction $f=1$. For comparison, Fisher et al. (2012) found slightly worse results: $\rho=0.97, a=0.94, f=0.9$ (right panel). It should be noted that the MEF method (Longcope 2004) also accurately recon- structed the total Poynting flux in the tests by Welsch et al. (2007), but the spatial correlation between the true and reconstructed fluxes was significantly worse at $\rho=0.85$ (see their Figure 14). Decomposing the total Poynting flux into potential and free components (see Section 3.1), we find that the PDFI reconstructs $100 \%$ of both the potential and free components $(f=1)$ and the slope between the reconstructed and PDFI is one $(a=1)$. The free and potential components comprise $87 \%$ and $13 \%$ of the total unsigned Poynting flux, respectively.

We also test how sensitive the Poynting fluxes are to errors in the vertical Doppler velocity. We find that if there is a random Doppler velocity noise of $0.05 \mathrm{~km} \mathrm{~s}^{-1}$ amplitude $\left(\bar{v}_{z}=0\right)$, i.e., around 10 to $20 \%$ of the signal, then it does not significantly affect the Poynting flux: the error increases slightly from 0.14 to 0.15 , the slope remains close to one $(a=0.98)$, and the fraction $f=1.0$. However, if there is a bias Doppler velocity that increases all the velocities by $0.05 \mathrm{~km} \mathrm{~s}^{-1}$ (toward the viewer) $\left(\bar{v}_{z}=0.05\right)$, then the slope and the fraction increase to $a=1.2$ and the error is Err. $=0.2$. Similarly, if all velocities 

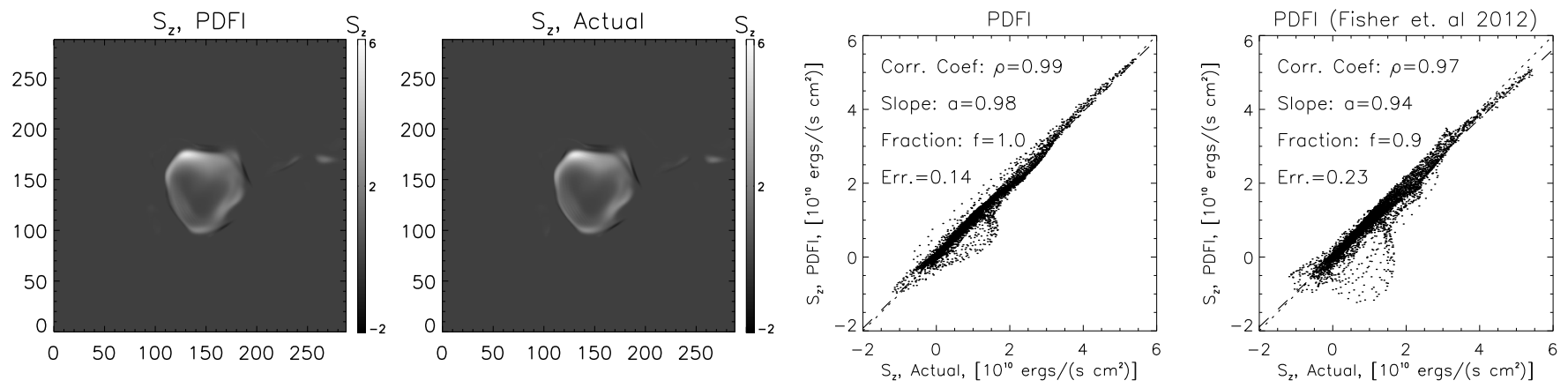

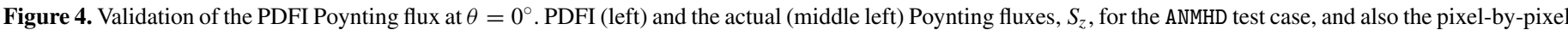

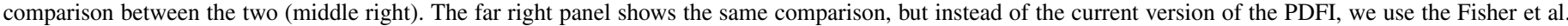
(2012) method.
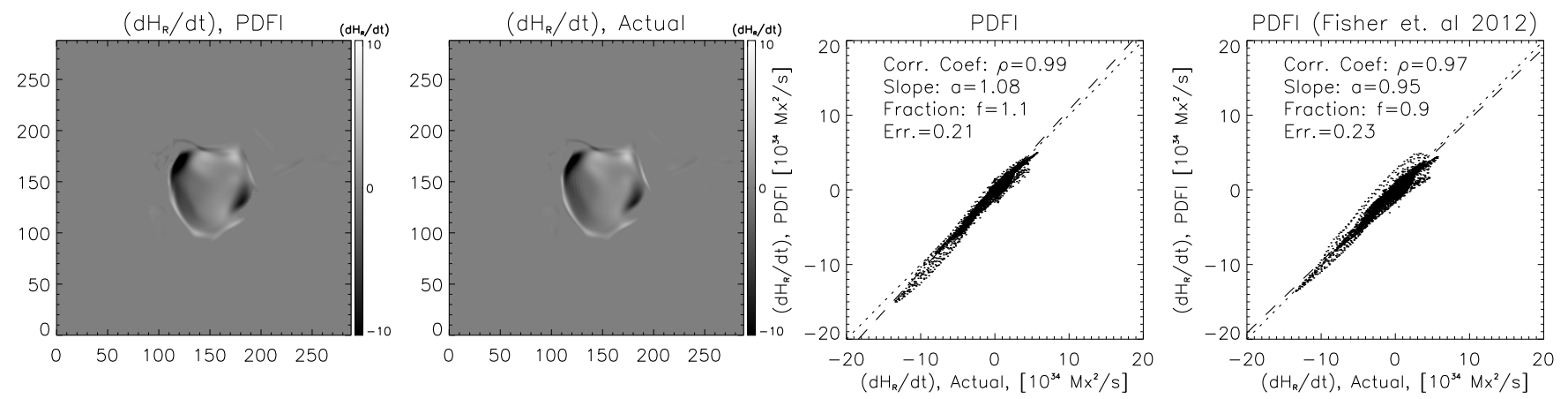

Figure 5. Validation of the PDFI helicity flux at $\theta=0^{\circ}$. See caption of Figure 4.
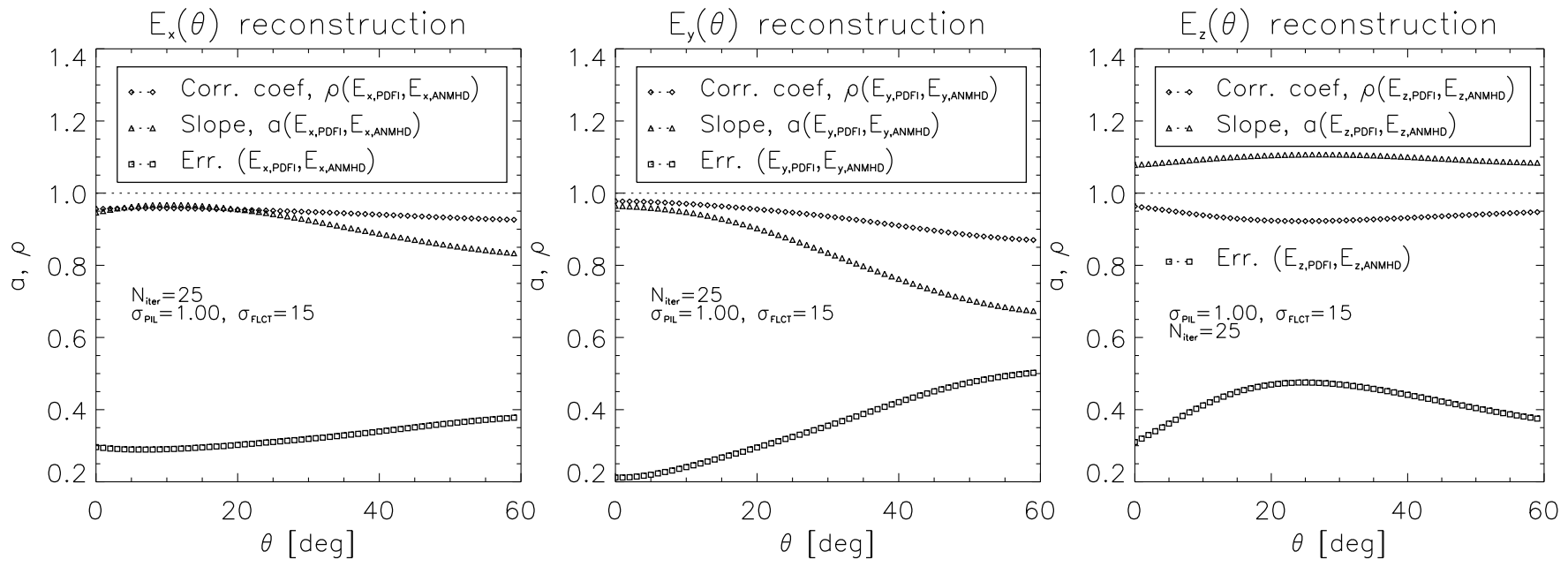

Figure 6. Validation of PDFI electric fields at viewing angles in the range of $\theta=\left[0^{\circ}, 60^{\circ}\right]$ : quality of $E_{x}$ (left), $E_{y}$ (middle) and $E_{z}$ (right) reconstructions.

decrease by $0.05 \mathrm{~km} \mathrm{~s}^{-1}\left(\bar{v}_{z}=-0.05\right)$, then the slope decreases to $a=0.8$ and the fraction to $f=0.8$. This test demonstrates how important it is to remove the Doppler velocity bias (Welsch et al. 2013) when inferring electric fields from the observations.

In Figure 5, we compare actual ANMHD and the PDFI helicity flux rates calculated from $\mathbf{E}$. We find a good agreement between the two: the correlation coefficient $\rho=0.99$, the slope $a=$ 1.08 , the fraction $f=1.1$, and the error Err. $=0.21$. For comparison, using the Fisher et al. (2012) electric fields, we find a very similar helicity flux rate but with a slightly larger scatter $(E r r .=0.23): \rho=0.97, a=0.95, f=0.9$. The differences between our approach here and that of Fisher et al. (2012) are the adoption of the FISHPACK software to solve the two-dimensional Poisson equations, the ability to compute contributions to Doppler-shift electric fields from non-normal viewing angles and a much more systematic and quantitative testing of the accuracy and robustness of the technique and its parameters.

\subsection{Quality Of Electric Field and Poynting Flux Reconstructions At Non-Zero Viewing Angles}

To test performance of PDFI method at non-normal viewing angles $\theta$, we calculated the electric field (Figure 6) and Poynting fluxes (Figure 7) at values of $\theta$ ranging from 0 to 60 degrees.

Figure 6 shows that at $\theta=0^{\circ}$, the error between reconstructed and the actual ANMHD electric fields is the smallest and as the angle increases, the quality of the reconstruction decreases. For $\theta<30^{\circ}$, for all $\mathbf{E}$ components, PDFI correctly identifies the slope between reconstructed and the actual ANMHD electric fields within a $10 \%$ difference. At the largest angle, $\theta=60^{\circ}$, the slope is $a=0.83$ for $E_{x}$ and $a=0.67$ for $E_{y}$, and the error in 

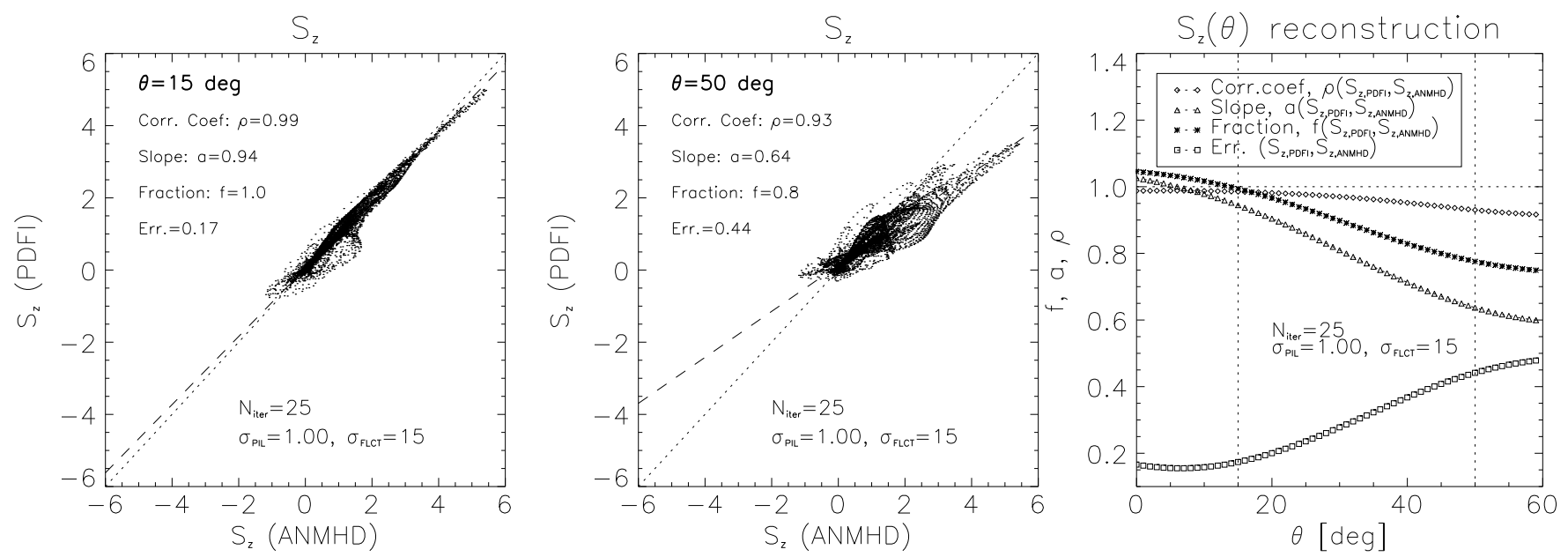

Figure 7. Validation of PDFI Poynting fluxes at viewing angles in the range of $\theta=\left[0^{\circ}, 60^{\circ}\right]$. Left and middle: pixel-to-pixel scatter plots comparing actual and PDFI Poynting fluxes at viewing angles $\theta=15^{\circ}$ (left) and $\theta=50^{\circ}$ (right). Right: quality of $S_{z}$ reconstructions in the range of $\theta=\left[0^{\circ}, 60^{\circ}\right]$.

Table 3

Comparison of $\left[E_{x}, E_{y}, E_{z}, S_{z},\left(d H_{R}\right) /(d t)\right]$, Derived using Different Electric Field Inversion Techniques (1-10), with the Actual ANMHD Quantities

\begin{tabular}{|c|c|c|c|c|c|}
\hline All & $E_{x}$ & $E_{y}$ & $E_{z}$ & $S_{z}$ & $\left(d H_{R} / d t\right)$ \\
\hline (1) $\mathrm{P}$ & ${ }^{0.57} 0.26_{0.83}$ & $0.780 .51_{0.64}$ & $0.931 .10_{0.44}$ & $0.780 .40_{0.68}$ & ${ }^{0.860 .53_{0.57}}$ \\
\hline (2) PI & ${ }^{0.88} 0.66_{0.59}$ & ${ }^{0.87} 0.630 .50$ & ${ }^{0.96} 1.06_{0.43}$ & ${ }^{0.92} 0.60_{0.42}$ & ${ }^{0.95} 0.93 \quad 0.34$ \\
\hline (3) PFI & ${ }^{0.91} 0.78_{0.42}$ & ${ }^{0.87} 0.630 .51$ & ${ }^{0.96} 1.06_{0.32}$ & 0.920 .590 .48 & ${ }^{0.96} 0.94_{0.28}$ \\
\hline (4) PDI & ${ }^{0.93} 0.83 \quad 0.37$ & ${ }^{0.97} 0.96_{0.24}$ & ${ }^{0.96} 1.070 .32$ & 0.981 .030 .19 & ${ }^{0.98} 1.090 .25$ \\
\hline (5) PDFI & ${ }^{0.97} 0.94_{0.25}$ & 0.980 .930 .19 & ${ }^{0.96} 1.070 .31$ & ${ }^{0.99} 0.98_{0.14}$ & ${ }^{0.99} 1.08 \quad 0.21$ \\
\hline (6) PDFI (Fisher) & ${ }^{0.91} 0.830 .41$ & $0.970 .94_{0.25}$ & ${ }^{0.96} 1.05_{0.31}$ & ${ }^{0.97} 0.94_{0.23}$ & ${ }^{0.97} 0.950 .23$ \\
\hline (7) $\operatorname{PDFI}\left(\theta=30^{\circ}\right)$ & ${ }^{0.95} 0.930 .32$ & ${ }^{0.94} 0.830 .36$ & ${ }^{0.92} 1.11_{0.47}$ & ${ }^{0.97} 0.810 .28$ & ${ }^{0.96} 1.080 .33$ \\
\hline (8) FI & $0.680 .49_{0.73}$ & $0.640 .36_{0.78}$ & ${ }^{0.84} 0.68_{0.54}$ & $0.330 .09_{0.95}$ & $0.850 .61_{0.55}$ \\
\hline (9) $\mathrm{DI}\left(\theta=0^{\circ}\right)$ & ${ }^{0.52} 0.350 .86$ & $0.820 .68_{0.57}$ & $---\ldots$ & ${ }^{0.94} 0.920 .34$ & ${ }^{0.73} 0.40_{0.71}$ \\
\hline (10) DFI $\left(\theta=0^{\circ}\right)$ & ${ }^{0.91} 0.84_{0.41}$ & ${ }^{0.97} 1.04_{0.26}$ & ${ }^{0.84} 0.680 .54$ & 0.961 .020 .28 & ${ }^{0.97} 1.01 \quad 0.25$ \\
\hline
\end{tabular}

Notes. Each entry in the table has a form of [ ${ }^{\rho} a_{E r r}$. $]$, where slope $a$, correlation coefficient $\rho$ and Err. are described in Section 4.1. The ideal reconstruction satisfies $\left[{ }^{\rho} a_{E r r}\right]=\left({ }^{1.00} 1.00_{0.00}\right)$. For a plot, see Figure 10.

these variables reaches up to $50 \%$. In contrast to the horizontal electric field, the vertical component $E_{z}$ is relatively insensitive to the viewing angle: the slope $a$ varies within $[1.08,1.10]$. The latter is not surprising since the Doppler contribution, which has most of the angular dependence, primarily constrains the horizontal field. For all $\mathbf{E}$ components, the correlation coefficient at $\theta=[0,60]^{\circ}$ is quite high, $\rho>0.9$, implying that the estimates of the slope shown on the plot adequately describe the quality of the reconstruction.

Figure 7 describes the quality of the vertical Poynting flux reconstruction at different viewing angles. Two left panels show a point-to-point comparison between the ANMHD and PDFI Poynting fluxes at $\theta=15^{\circ}$ (left panel) and $\theta=50^{\circ}$ (middle panel). At $\theta=15^{\circ}$, the agreement between the ANMHD and PDFI $S_{z}$ is very good: the slope $a=0.94, \rho=0.99, f=1, E r r .=$ 0.17 . Increasing the angle, at $\theta=50^{\circ}$ the scatter increases and the slope decreases to $a=0.64$, the error $E r r$. $=0.44$, and PDFI method recovers $80 \%$ of the total actual flux. The right panel summarizes the quality of the Poynting flux reconstruction for viewing angles within the $\left[0,60^{\circ}\right]$ range. At close-to-normal angles, $\theta<20^{\circ}$, PDFI recovers more than $95 \%$ of the total energy flux and the error between reconstructed and the actual Poynting fluxes is less than $15 \%(E r r .=0.15)$. At larger angles, $\theta<60^{\circ}, 75 \%$ of the total flux is recovered and the error Err. $<0.5$. For the helicity flux rate, at $\theta<60^{\circ}$, more than $90 \%$ of the total flux is recovered correctly and the error Err. $=0.3$.

\subsection{Comparison Of Electric Field Reconstruction Techniques}

In this section, we analyze roles that the inductive and noninductive components play in reconstructed vertical Poynting flux (Figure 8), electric field and helicity fluxes (Figures 9 and 10, Table 3).

Figure 8 shows scatter plots comparing the actual vertical Poynting flux with the Poynting fluxes derived with different reconstruction methods: (1) P, (2) PI, (3) PFI, (4) PDI, (5) PDFI, (6) PDFI at non-normal angle $\theta=30^{\circ}$, (7) FI, (8) DI, and (9) DFI. The nomenclature that we use here is described in Section 2.3.4 and Table 1. Using just the inductive part of the electric field $(\mathrm{P})$, we reconstruct only $40 \%$ of the total Poynting flux and the scatter from the linear dependence is large $($ Err. $=0.68)$. Adding the ideal MHD assumption (PI) adds $30 \%$ more Poynting flux, leading to much less scatter (larger $\rho)$ and a smaller error $(E r r .=0.48)$. Inclusion of the nonideal contribution due to horizontal plasma velocities, inferred from the FLCT (PFI), does not improve the solution. However, when we include the Doppler non-inductive component alone (PDI), we reconstruct $100 \%$ of the flux, i.e., the role of the Doppler contribution is much higher than that from the FLCT horizontal velocities. Finally, when we add both the 

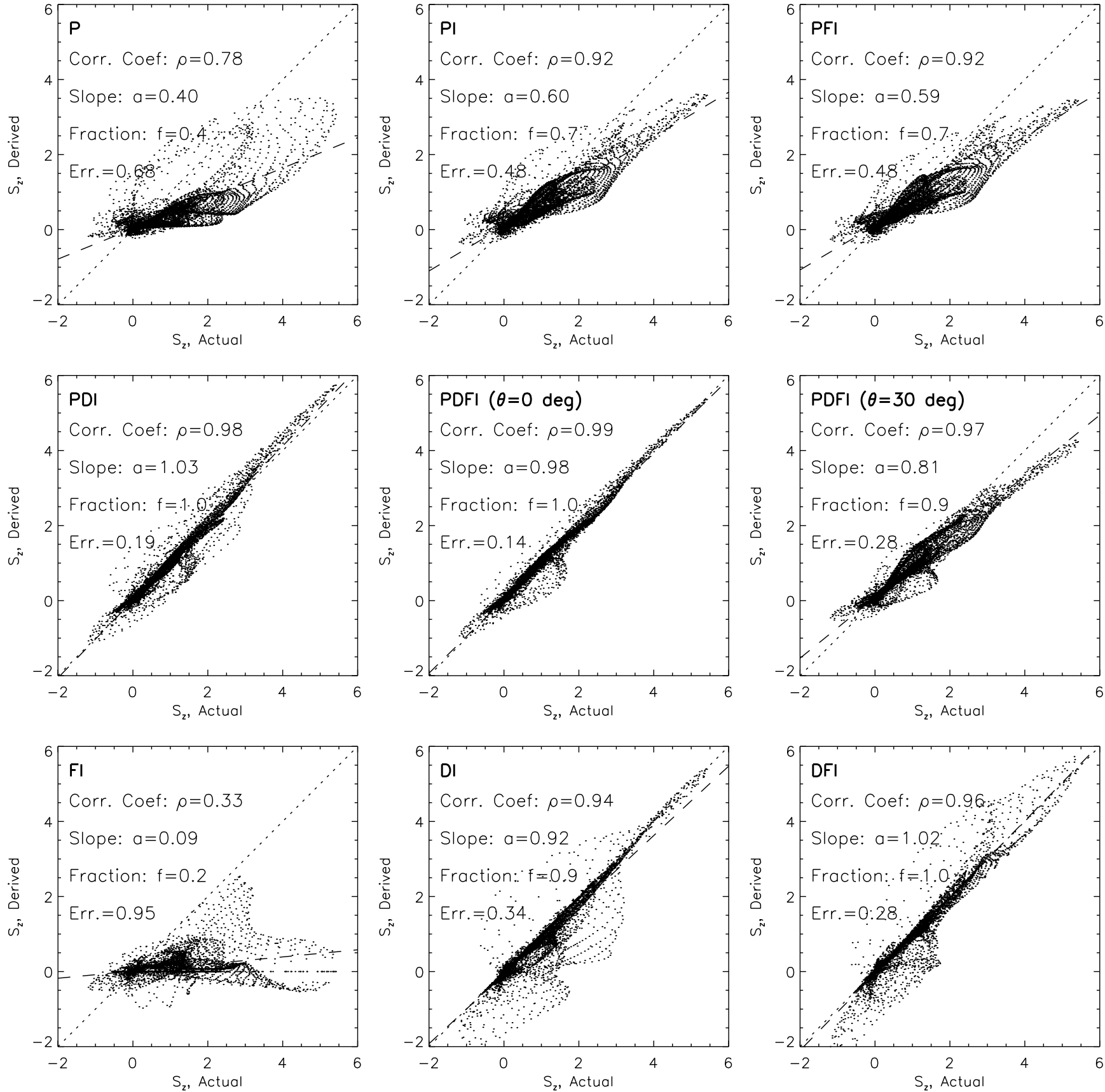

Figure 8. Comparison of the derived and the actual ANMHD vertical Poynting fluxes for different electric field inversion methods. Each method's results are shown in a separate panel with method's name indicated in the upper left corner and described in Section 2.3.4.

FLCT and the Doppler contributions (PDFI), the final Poynting flux is the closest to the actual $S_{z}$ with a slightly smaller error $(E r r .=0.14)$ than in the PDI case $(E r r .=0.19)$. On a separate note, if we use a non-PTD ideal inversion technique instead of the PTD, $\mathbf{E}=-\mathbf{V} \times \mathbf{B}, V_{z}=0$ (FI), we reconstruct only $20 \%$ of the flux, i.e., the agreement between the reconstruction and the actual $S_{z}$ is poor $(E r r$. $=0.95)$. If we know the vertical Doppler velocity (DI), then $90 \%$ of the actual flux is reconstructed and with a good agreement $(\rho=0.94, a=0.92, f=0.9, E r r .=0.34)$. With both vertical and horizontal velocities (DFI), we extract $100 \%$ of the total Poynting flux, the slope $a=1.02$, and the error $E r r$. $=0.28$. How is this different from $S_{z}$ from the PDFI?
The PDFI electric field that includes both inductive and noninductive contributions yields a factor of two smaller error in $S_{z}(E r r .=0.14)$ than the DFI field and shows less scatter, especially in the regions of strong Poynting fluxes, thus it better represents the vertical Poynting flux. For completeness, in Figure 9 we also compare helicity flux rates, $\left(d H_{R}\right) /(d t)$, from different reconstruction methods with the actual helicity flux rate. We remark that the crucial role the Doppler signal plays in reconstructing the Poynting and helicity fluxes in this case might be due to the process being modeled in the ANMHD simulation: an emerging magnetic bipole. It is possible that Doppler inputs to electric field estimates are less important when flux is not actively emerging. 

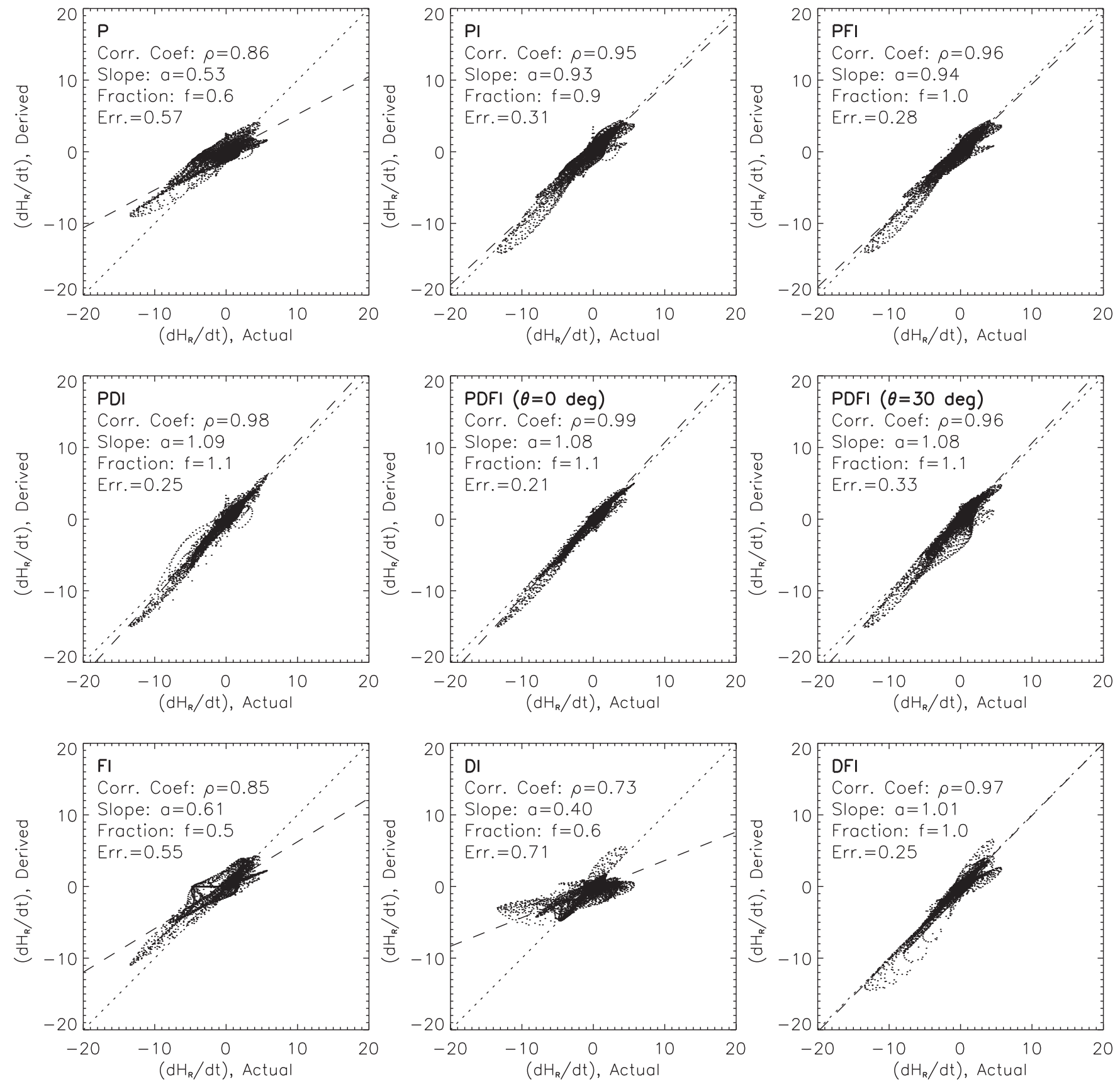

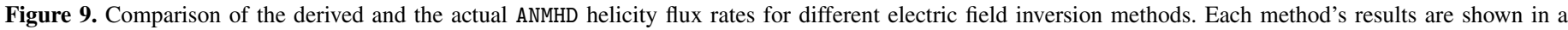
separate panel with method's name indicated in the upper left corner and described in Section 2.3.4.

In Figure 10 and Table 3, we summarize the quality of different electric field inversion techniques in the same way we did for $S_{z}$ and $\left(d H_{R}\right) /(d t)$ for all the variables, $\left[E_{x}, E_{y}, E_{z}, S_{z},\left(d H_{R}\right) /(d t)\right]$, where $\left[E_{x}, E_{y}, E_{z}\right]$ are the three components of the electric field, $S_{z}$ is the vertical Poynting flux, and $\left(d H_{R}\right) /(d t)$ is the helicity flux rate (see Equations (41) and (42)). Besides the methods shown previously (see Figure 8), we also add the method number 6, "PDFI (Fisher)," which compares variables reconstructed in Fisher et al. (2012) with the actual ANMHD values.

The $\mathrm{P}$ solution yields the worst reconstructions-the slope between the actual and derived $E_{x}$ and $E_{y}$ is 0.26 and 0.51 , respectively, and the correlation coefficient in both cases is less than 0.8 . The quantity $E_{z}$, however, is reconstructed very well-the slope is 1.10 and the correlation coefficient is 0.93 . Adding the other ingredients only slightly improves the slope and the correlation coefficient of $E_{z}$. The Poynting and helicity fluxes, $S_{z}$ and $\left(d H_{R}\right) /(d t)$, respectively, are reconstructed poorly: the slopes are 0.4 and 0.53 , respectively.

Application of the ideal MHD constraint to the inductive solution (PI, case 2) significantly improves reconstruction of $E_{x}$ and $E_{y}$ : the slope for $E_{x}$ increases from 0.26 to 0.66 and from 0.51 to 0.63 for $E_{y}$. This results in much better quality of the Poynting and helicity fluxes reconstructions: the slope for the 

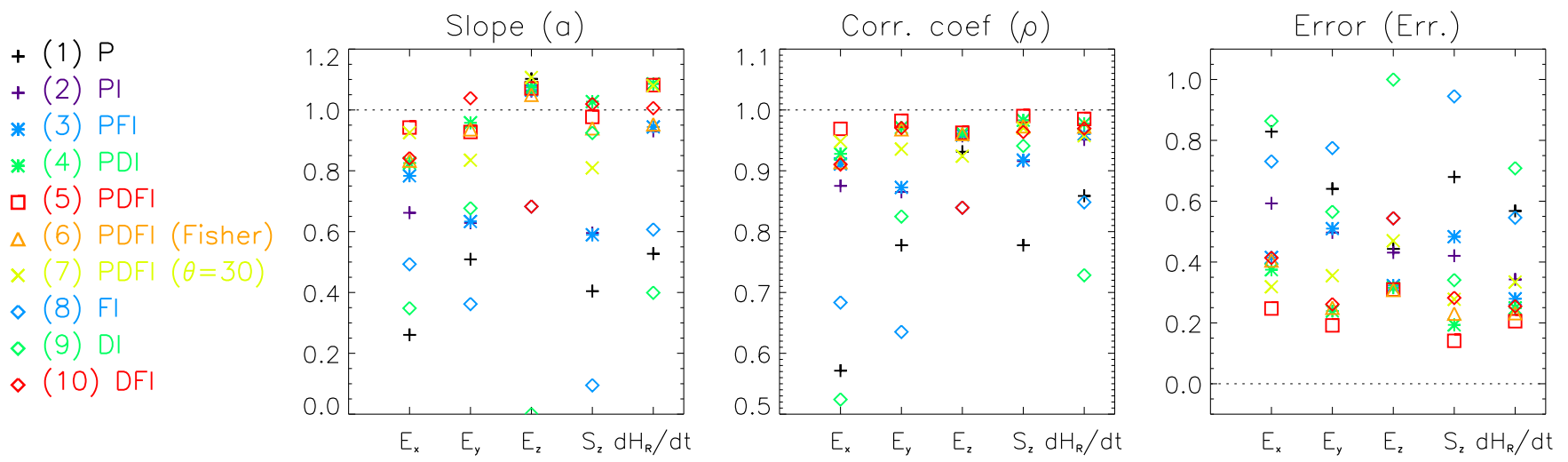

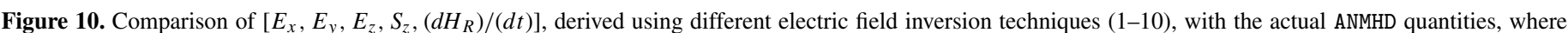

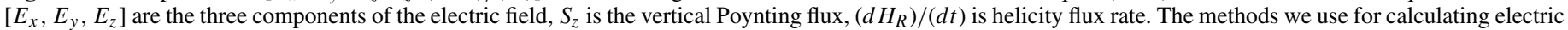

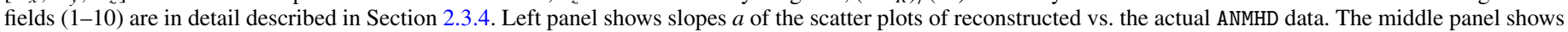

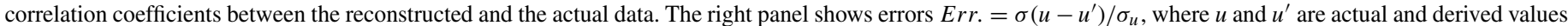

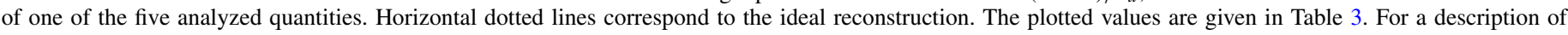
[a, $\rho, E r r$. ], see Section 4.1.

(A color version of this figure is available in the online journal.)

Poynting flux changes from 0.4 to 0.6 from $\mathrm{P}$ to PI solution and from 0.53 to 0.93 for helicity flux. The error decreases by more than $20 \%$.

Addition of the non-inductive contribution from horizontal velocities (PFI, case 3), slightly improves the slope and correlation coefficient of $E_{x}$ : the slope changes from 0.66 to 0.78 and from 0.88 to 0.91 for the correlation coefficients. The error decreases as well.

Inclusion of the non-inductive contribution from the vertical Doppler velocity, (PDI, case 4), provides much better improvement than the FLCT contribution (PFI; Fisher et al. 2012). The slopes and correlation coefficients for both $E_{x}$ and $E_{y}$ increase significantly from 0.66 and 0.63 for the PI solution to 0.82 and 0.96 , respectively. This results in much better reconstruction of $S_{z}$ : the slope for $S_{z}$ changes from 0.6 to 1.03 . The errors, especially for $E_{y}$, decrease, change from 0.51 to 0.24 .

Finally, in the PDFI case (case 5) we add both non-inductive, FLCT, and Doppler contributions. We find that the PDFI method yields the best agreement with the ANMHD variables. The slopes for $E_{x}$ and $E_{y}$ are 0.94 and 0.93 with the correlation coefficients of 0.97 and 0.98 , respectively. The Poynting and helicity fluxes have slopes of 0.98 and 1.08 with correlation coefficients of 0.99 and 0.99. The error varies from 0.14 (for $S_{z}$ ) to 0.31 (for $E_{z}$ ). To summarize, in terms of the fractions, PDFI method predicts roughly $100 \%$ of the Poynting flux and $110 \%$ of the helicity flux rate. It yields slopes and correlation coefficients that are similar to Fisher et al. (2012; PDFI (Fisher), see case 6), but has smaller errors (compare 0.14 versus 0.23 for $S_{z}$ ).

At a non-normal viewing angle of $\theta=30^{\circ}$ (case 7), the agreement gets slightly worse, but still the slopes are close to one and the error, for example, in the Poynting flux is $28 \%$. We reconstruct $90 \%$ of the total Poynting flux and $110 \%$ of the total helicity flux. Applying the same technique (Section 2.3.3) at normal viewing angle we find the same results as the ones derived using the normal viewing angle technique (Section 2.3.1).

We also calculate $\mathbf{E}, S_{z}$ and $d H_{R} / d t$ using the ideal non-PTD $\mathbf{E}=-\mathbf{V} \times \mathbf{B}$ formalism: the FI, DI, and DFI (cases 8, 9, and 10). If only horizontal components of the velocity field are used and $V_{z}$ is set to zero (FI), then we get the worst reconstruction- the slopes for $E_{x}$ and $E_{y}$ are less than 0.5. The slope for the Poynting flux is $a=0.09$ and the fraction $f=0.5$. This is much worse than the PFI solution: $a=0.95$ and $f=1.0$. The difference between PFI and FI solutions might seem surprising at first, since both PFI and FI use the same information on the input. However, while PFI solves the induction equation, the FI method does not. When we take the vertical velocity into account (DI), the quantity $E_{z}$ is unknown and the quality of $E_{x}$ and $E_{y}$ improves slightly, raising from slopes of $a=0.49$ and $a=0.36$ to $a=0.35$ and $a=0.68$, respectively. As a result, the reconstruction of $S_{z}$ improves from $a=0.09$ to $a=0.92$ and the fraction is $f=0.9$. This is slightly worse than the $S_{z}$ from PDI: $a=1.03$ and $f=1.0$. For the non-inductive field estimates, we get the best agreement for $S_{z}$ when both horizontal and Doppler velocities are taken into account (DFI, case 10): $a=1.02, f=1.0$. (See also Ravindra et al. 2008.) Comparing the DFI with the PDFI reconstruction, we find that DFI is able to capture $E_{y}$, Poynting, and helicity fluxes, although with larger errors than the PDFI, while $E_{x}$ and $E_{z}$ yield a poor reconstruction. In contrast, PDFI restores all three components of the electric field correctly, as well as the Poynting and helicity fluxes, and with smaller errors. The reconstructions that include PTD (PI, PFI, PDI) also do a much better job than non-PTD solutions (FI, DI) when only one contribution to the velocity field (Doppler or FLCT) is available.

We separately compare actual and calculated helicity flux rates and estimate the quality of the helicity flux rate reconstruction when only velocity estimates are used (FI or DI, see Equation (42)) instead of the electric field (PI, PDI, PFI). When only the horizontal velocity field has been estimated (PFI or FI), then PFI yields much better reconstruction of helicity flux than FI: $a=0.95, f=1.0, E r r$. $=0.28$ (PFI) versus $a=0.61, f=0.5, E r r .=0.55(\mathrm{FI})$. When only the Doppler velocity field is known (PDI or DI), then PDI also does a much better job than DI: $a=1.08, f=1.1, E r r .=0.25$ (PDI) versus $a=0.4, f=0.6, E r r .=0.71$ (DI). Finally, if both horizontal and Doppler fields are known (PDFI or DFI), then both PDFI and DFI get similar values of helicity fluxes. However, the PDFI solution gets slightly better correlation coefficients and smaller errors and hence less scatter.

Figure 10 summarizes performance of all the methods in three panels (slope, correlation coefficient, and error). For the 
Table 4

Comparison of Accuracy of Poynting and Rate of Relative Helicity Fluxes Estimates between the PDFI, PFI, DAVE+ANMHD, and DAVE4VM (Schuck 2008) over $\left|B_{z}\right|>370 \mathrm{G}$

\begin{tabular}{l|cccc|cccc}
\hline \hline & PFI & DAVE4VM & PDFI & DAVE+ANMHD & PFI & DAVE4VM & PDFI & DAVE+ANMHD \\
\hline & \multicolumn{9}{|c|}{ Poynting flux, $S_{z}$} \\
Slope, $a$ & 0.92 & 0.71 & 0.99 & 0.99 & 0.96 & 0.9 & 0.99 \\
Corr. coef., $\rho$ & 0.59 & 0.83 & 0.98 & 0.96 & 0.95 & 0.94 & 1.08 \\
Fraction, $f$ & 0.7 & 0.76 & 1.0 & 0.99 & 1.0 & 0.94 & 1.1 \\
\end{tabular}

Notes. An ideal reconstruction satisfies $a=1, \rho=1, f=1$.

PDFI method, shown with red squares, we get the most accurate electric fields and Poynting and helicity fluxes. The PDFI method outperforms ideal non-PTD methods (FI, DI, DFI).

\section{DISCUSSION AND CONCLUSIONS}

We have modified the methods described in Fisher et al. (2012) for estimating electric fields from vector magnetic fields and Doppler velocities to incorporate non-normal viewing angles and the faster, more robust FISHPACK solver to produce a PTD-Doppler-FLCT-Ideal-Ohm's solver (PDFI) that could be easily applied to observed data. We then used a pair of synthetic magnetograms extracted from MHD simulations in which magnetic and Doppler velocity fields are known to estimate the electric fields. Finally, we characterized the accuracy of the derived electric fields and the fluxes of magnetic energy and helicities. In this section, we summarize the strengths and weaknesses of the PDFI technique, compare its results with other techniques, and describe how it will be used to analyze HMI vector magnetograms and Doppler data.

At zero viewing angle, the accuracy of the PDFI method is excellent. Using the PDFI method, the total Poynting flux, $S_{z}$, is estimated with an error of less than $1 \%$ and the total helicity flux rate, $\left(d H_{R}\right) /(d t)$, with an error of $10 \%$. The rms of the integrands are $12 \%$ and $26 \%$, respectively. These estimates are more accurate and much faster to derive than those from Fisher et al. (2012; see Figures 4 and 5 and Table 3). ${ }^{1}$

With increasing viewing angle $\theta$, the accuracy of the PDFI method slowly decreases. At $\theta<20^{\circ}$, PDFI recovers more than $95 \%$ of the total Poynting flux with a slope of $a=0.98$. At $\theta<60^{\circ}$, PDFI recovers, more than $75 \%$ of $S_{z}$ with the slope of $a=0.6$ (see Figure 7). As for the helicity flux rate, at $\theta<60^{\circ}$, more than $90 \%$ of the total $\left(d H_{R}\right) /(d t)$ is recovered with the slope of $a=1.0$.

In this paper, we compare the quality of reconstructions of electric fields, Poynting and helicity fluxes from the PTD-based methods (PFI, PDI, PDFI) that explicitly enforce consistency of electric fields with evolution of $\mathbf{B}$ with non-PTD methods (FI, DI, DFI), such as, e.g., FLCT, that derive horizontal velocities by tracking the vertical magnetic field. We show that when both horizontal and Doppler velocity fields are known, the two approaches, PDFI and DFI, yield similar results for helicity and Poynting fluxes, with the PTD-based PDFI method having slightly smaller errors. However, when either the Doppler or the FLCT velocity field is unknown, the PTD methods are much better in reconstructing both helicity and Poynting fluxes than non-PTD methods. For example, when only the

\footnotetext{
1 For the Poynting flux, the slope between the actual and the PDFI $S_{z}$ is $a=0.98$ in this paper versus $a=0.94$ in Fisher et al. (2012) and for the helicity flux rate, the slope $a=1.08$ in this paper versus $a=0.95$ in Fisher et al. (2012). In both cases the scatter in the PDFIs is smaller than in Fisher et al. (2012).
}

horizontal velocity is known, PFI reconstructs $70 \%$ of total $S_{z}$ and $100 \%$ of total $\left(d H_{R}\right) /(d t)$ while FI reconstructs only $20 \%$ and $50 \%$, respectively. Similarly, when only the Doppler velocity is known, then PDI reconstructs $100 \%$ of total $S_{z}$ and $110 \%$ of total $\left(d H_{R}\right) /(d t)$, while DI reconstructs $90 \%$ and $60 \%$, respectively.

For the helicity flux rate, our results imply that in order to correctly capture the helicity flux rate, one cannot only use the velocity field determined from the tracking of the vertical magnetic field (FI), but instead must separately include the emergence term due to vertical velocity: the Démoulin \& Berger (2003) conjecture does not apply here, consistent with conclusions of Schuck (2008), Liu \& Schuck (2012), and Ravindra et al. (2008). We note again that the ANMHD data we analyze were drawn from a simulation of an emerging magnetic bipole, a configuration in which vertical flows (used as our Doppler velocity input) play particularly strong roles in the fluxes of magnetic energy and helicity. This might not be true in active regions when substantial amounts of new flux are not emerging.

To put our results into context, in Table 4, we compare the PDFI Poynting and helicity fluxes that we derive in this paper to those calculated with DAVE4VM and DAVE+ANMHD (Schuck 2008). While DAVE4VM predicts roughly $75 \%$ of the total Poynting flux and $95 \%$ of the helicity flux rate, the PDFI method has a better performance with less than $1 \%$ error in the total Poynting flux and a $10 \%$ error in the helicity flux rate. One should keep in mind, however, that DAVE4VM, unlike PDFI, does not take the Doppler velocity into account, hence it is fair to compare not the PDFI's, but instead the PFI's and DAVE4VM's results. When we do that we find that the PFI performs similarly to DAVE4VM and that both miss more than $25 \%$ of the total Poynting flux. In contrast, PDFI, which includes both horizontal and Doppler velocities, yields an excellent agreement with the actual ANMHD quantities (see Table 4). Note that the PDFI estimate for helicity flux is better than that of the DAVE+ANMHD, which takes the Doppler signal into account (see Figure 14 in Schuck 2008). In addition, we remark that while the MEF method (Longcope 2004) performed well in the tests by Welsch et al. (2007), here we find the PDFI method to be superior by several statistical measures for the same test data (compare Figure 14 in Welsch et al. 2007 with Figure 8).

To estimate the speed of the PDFI method, we did a series of inversion runs for the ANMHD data set on a MacBook Pro laptop with 2GHz Intel Core i7 Processor and 8 GB 1333 MHz DDR3. For this test case where $N_{x}=288$ and $N_{y}=288$ pixels, it takes 0.24 seconds to calculate the plain P electric field, 1.2 seconds for the PDF electric field, and 7.3 seconds for the ideal PDFI electric field ( $N_{\text {iter }}=25$; see Figure 2).

One of the major weaknesses of the PDFI method, and also of any technique that uses the Doppler data, is a strong dependence 
on the Doppler bias velocity (see Section 4.2). In this paper, to calculate PDFI electric fields, we assumed that we know the Doppler velocity field. In reality, however, the observed LOS velocity has a bias due to instrumental variations and the known correlation between the intensity and blueshift, known as convective blueshift. Recently, Welsch et al. (2013) addressed this issue by presenting several methods to estimate the absolute calibration of LOS velocities in solar active regions near the disk center that we apply to the HMI observations of the active region NOAA 11158 (M. D. Kazachenko et al. 2014, in preparation).

Another weakness of the PDFI method is the absence of clear understanding of the extent to which additional information from other sources (besides Doppler and horizontal velocities) is necessary to fully specify the non-inductive part of the electric field. In the past, Fisher et al. (2012) showed that the Doppler signal near PILs and horizontal velocities away from the PILs contain important non-inductive information for the electric field that cannot be derived from the Faraday's law. In this paper, we tested this approach at non-zero viewing angles and found a good agreement for viewing angles of less than $50^{\circ}$ for the ANMHD test case. There may be additional degrees of freedom for the non-inductive electric field that are not fully captured by the Doppler plus transverse magnetic field fluxemergence contribution described in Fisher et al. (2012). For example, rigid rotations of magnetic structures (e.g., sunspot rotation) that include a high degree of symmetry will have electric field components that are not fully captured by the PDFI formalism because of the lack of change they produce in the vector magnetic field on the photosphere. However, such motions transport significant energy and helicity into the corona (Kazachenko et al. 2009). For this reason, other tests of the emergence of a twisted flux tube from the interior into the solar atmosphere with different subsurface twisted flux tube configurations should be done. These tests will allow us formulate and then to incorporate any necessary additional electric fields corresponding to such horizontal vortical motions into the PDFI solution using observational information, such as observed sunspot penumbral motions, as input. Furthermore, Doppler observations from a non-normal viewing angle can also be used to capture such horizontal motions and help to recover the corresponding electric fields.

We thank the US taxpayers for providing the funding that made this research possible. We thank Mark Cheung and the anonymous referee for their thoughtful input that has improved the manuscript. We acknowledge funding from the Coronal Global Evolutionary Model (CGEM) award NSF AGS 1321474 (M.D.K., B.T.W., G.H.F.), NSF Award AGS-1048318 (G.H.F.), NASA Award NNX13AK54G (M.D.K.), NSF SHINE Postdoc Award 1027296 (M.D.K.), NSF's National Space Weather Program AGS-1024862 (B.T.W.), the NASA Living-With-aStar TR\&T Program NNX11AQ56G (M.D.K., B.T.W., G.H.F.), and the NASA Heliophysics Theory Program NNX11AJ65G (G.H.F., B.T.W.).

\section{APPENDIX}

\section{GENERALIZATION OF PTD FORMALISM TO SPHERICAL COORDINATES}

We can extend the PTD formalism from Cartesian to spherical coordinates and show that the form of the equations we need to solve to find the electric field vector is the same in both coordinate systems.
Similar to the Cartesian case (see Equations (1)-(5)), we decompose the magnetic field vector $\mathbf{B}=\left(B_{r}, B_{\theta}, B_{\phi}\right)$ into two "poloidal" and "toroidal" potentials, $\mathcal{B}$ and $\mathcal{J}: \mathbf{B}=$ $\nabla \times \nabla \times \mathcal{B} \hat{\mathbf{r}}+\nabla \times \mathcal{J} \hat{\mathbf{r}}$ (Moffatt 1978). We then take a partial time derivative of $\mathbf{B}$ and express the $(\nabla \times)$ operator in spherical coordinates:

$$
\begin{aligned}
\dot{\mathbf{B}}= & \nabla \times \nabla \times \dot{\mathcal{B}} \hat{\mathbf{r}}+\nabla \times \dot{\mathcal{J}} \hat{\mathbf{r}} \\
= & -\nabla^{2} \dot{\mathcal{B}} \hat{\mathbf{r}}+\nabla(\nabla \cdot \dot{\mathcal{B}} \hat{\mathbf{r}})+\nabla \times \dot{\mathcal{J}} \hat{\mathbf{r}} \\
= & \underbrace{\left[\frac{-1}{r^{2} \sin \theta} \frac{\partial}{\partial \theta}\left(\sin \theta \frac{\partial \dot{\mathcal{B}}}{\partial \theta}\right)-\frac{1}{r^{2} \sin ^{2} \theta}\left(\frac{\partial^{2} \dot{\mathcal{B}}}{\partial \phi^{2}}\right)\right]}_{-\nabla_{h}^{2} \dot{\mathcal{B}}} \hat{r}+ \\
& +\left[\frac{1}{r} \frac{\partial}{\partial \theta}\left(\frac{\partial \dot{\mathcal{B}}}{\partial r}\right)\right] \hat{\theta}+\left[\frac{1}{r \sin \theta} \frac{\partial}{\partial \phi}\left(\frac{\partial \dot{\mathcal{B}}}{\partial r}\right)\right] \hat{\phi}+\nabla \times \dot{\mathcal{J}} \hat{r} \\
= & -\nabla_{h}^{2} \dot{\mathcal{B}} \hat{r}+\nabla_{h}\left(\frac{\partial \dot{\mathcal{B}}}{\partial r}\right)+\nabla \times \dot{\mathcal{J}} \hat{r}
\end{aligned}
$$

By examining the $r$ component of the equation above, its horizontal divergence, and the $r$ component of its curl, we find three two-dimensional Poisson equations for the unknown functions $\dot{\mathcal{B}},(\partial \dot{\mathcal{B}}) /(\partial z)$ and $\dot{\mathcal{J}}$ :

$$
\begin{gathered}
-\dot{B}_{r}=\nabla_{h}^{2} \dot{\mathcal{B}}, \\
\nabla_{h} \cdot \dot{\mathbf{B}}_{h}=\nabla_{h}^{2}\left(\frac{\partial \dot{\mathcal{B}}}{\partial r}\right) \\
-\hat{r} \cdot\left(\nabla \times \dot{\mathbf{B}}_{h}\right)=\nabla_{h}^{2} \dot{\mathcal{J}},
\end{gathered}
$$

where the spherical form for the horizontal component of Laplace operator, $\nabla_{h}^{2}$, is shown above and the horizontal divergence operator, $\left(\nabla_{h} \cdot\right)$, has the standard expression for the divergence in spherical coordinates without the $r$-derivative term. Equations (A1)-(A3) can be solved with the HWSSSP subroutine in FISHPACK (Schwarztrauber 1974).

Uncurling Equation (A1) and comparing it with the Faraday's law, we derive the electric field $\mathbf{E}$ in terms of calculated $\dot{\mathcal{B}}$ and $\dot{\mathcal{J}}$ in spherical coordinates:

$$
c \mathbf{E}=c\left(E_{r}, E_{\phi}, E_{\theta}\right)=-\nabla \times \dot{\mathcal{B}} \hat{\mathbf{r}}-\dot{\mathcal{J}} \hat{\mathbf{r}}-\nabla \psi \equiv c \mathbf{E}^{\mathbf{P}}-\nabla \psi .
$$

\section{REFERENCES}

Abbett, W. P. 2007, ApJ, 665, 1469

Abbett, W. P., Fisher, G. H., \& Fan, Y. 2000, ApJ, 540, 548

Abbett, W. P., Mikić, Z., Linker, J. A., et al. 2004, JASTP, 66, 15

Berger, M. A. 1984, GApFD, 30, 79

Berger, M. A., \& Field, G. B. 1984, JFM, 147, 133

Berger, T. E., Schrijver, C. J., Shine, R. A., et al. 1995, ApJ, 454, 531

Chae, J., \& Sakurai, T. 2008, ApJ, 689, 593

Chandrasekhar, S. 1961, Hydrodynamic and Hydromagnetic Stability (New York: Dover),

Davis, W. D. 1977, SoPh, 54, 139

Démoulin, P., \& Berger, M. A. 2003, SoPh, 215, 203

Fan, Y., Zweibel, E. G., Linton, M. G., \& Fisher, G. H. 1999, ApJ, 521, 460

Finn, J., \& Antonsen, T. M., Jr. 1985, CoPPC, 9, 111

Fisher, G. H., \& Welsch, B. T. 2008, in ASP Conf. Ser. 383, Subsurface and Atmospheric Influences on Solar Activity, ed. R. Howe, R. W. Komm, K. S. Balasubramaniam, \& G. J. D. Petrie (San Francisco, CA: ASP), 373

Fisher, G. H., Welsch, B. T., \& Abbett, W. P. 2012, SoPh, 277, 153

Fisher, G. H., Welsch, B. T., Abbett, W. P., \& Bercik, D. J. 2010, ApJ, 715, 242

Hurlburt, N. E., Schrijver, C. J., Shine, R. A., \& Title, A. M. 1995, Helioseismology, Vol. 376 of ESA Special Publication (Noordwijk: ESA), 239 
Jordan, C., Bartoe, J.-D. F., \& Brueckner, G. E. 1980, ApJ, 240, 702

Kazachenko, M. D., Canfield, R. C., Longcope, D. W., et al. 2009, ApJ, 704, 1146

Kusano, K., Maeshiro, T., Yokoyama, T., \& Sakurai, T. 2002, ApJ, 577, 501

Lantz, S. R., \& Fan, Y. 1999, ApJ, 121, 247

Liu, Y., \& Schuck, P. W. 2012, ApJ, 761, 105

Longcope, D. W. 2004, ApJ, 612, 1181

Moffatt, H. K. 1978, Magnetic Field Generation in Electrically Conducting Fluids (Cambridge: Cambridge Univ. Press)

Moran, T., \& Foukal, P. 1991, SoPh, 135, 179

November, L. J., \& Simon, G. W. 1988, ApJ, 333, 427

Ravindra, B., Longcope, D. W., \& Abbett, W. P. 2008, ApJ, 677, 751
Scherrer, P. H., Schou, J., Bush, R. I., et al. 2012, SoPh, 275, 207

Schou, J., Scherrer, P. H., Bush, R. I., et al. 2012, SoPh, 275, 229

Schuck, P. W. 2006, ApJ, 646, 1358

Schuck, P. W. 2008, ApJ, 683, 1134

Schwarztrauber, P. 1974, JCoPh, 15, 46

Schwarztrauber, P. 1975, NCAR Tech. Note-TN, 138

Sweet, R. 1974, SJNA, 11, 506

Welsch, B. T. 2006, ApJ, 638, 1101

Welsch, B. T., Abbett, W. P., DeRosa, M. L., et al. 2007, ApJ, 670, 1434

Welsch, B. T., Fisher, G. H., Abbett, W. P., \& Regnier, S. 2004, ApJ, 610, 1148

Welsch, B. T., Fisher, G. H., \& Sun, X. 2013, ApJ, 765, 98

Wien, W. 1916, AnP, 354, 842 